\title{
AlBERT RAUGI \\ Théorème de la limite centrale pour un produit semi-direct d'un groupe de Lie résoluble simplement connexe de type rigide par un groupe compact
}

Publications des séminaires de mathématiques et informatique de Rennes, 1977, fascicule 3

«Séminaire de probabilités II », , p. 1-67

<http://www.numdam.org/item?id=PSMIR_1977__3_A9_0>

(C) Département de mathématiques et informatique, université de Rennes, 1977, tous droits réservés.

L'accès aux archives de la série « Publications mathématiques et informatiques de Rennes » implique l'accord avec les conditions générales d'utilisation (http://www.numdam.org/conditions). Toute utilisation commerciale ou impression systématique est constitutive d'une infraction pénale. Toute copie ou impression de ce fichier doit contenir la présente mention de copyright.

\section{Numdam}


THEOREME DE LA LIMITE CENTRALE POUR UN

PRODUIT SEMI-DIRECT D'UN GROUPE DE LJE RFSOLUBLE

SIMPLEMENT CONNEXE DF TYPE RIGIDE PAR UN GROUPE COMPACT

par Albert RAUGI

Soit $R$ un groupe de Lje résoluble connexe. Nous désignons par Ad la représentation adjointe de $R$. Nous disons que $R$ est de type rigide si les valeurs propres des éléments de Ad $R$ sont toutes de module égal à 1 .

Soit $G$ un produit semi-direct d'un groupe de Lie résoluble simplement connexe de type rigide par un groupe compact. Si $\lambda$ est une mesure de probabilité sur les boréliens de $G$, nous savons que $([1])$, lorsque $G n^{\prime e s t}$ pas compact, la $n^{\text {ième }}$ convolée, $\lambda^{n}$, de $\lambda$ converge vaguement vers zéro. Nous nous proposons de trouver une suite d'homéomorphismes $\left\{U_{n}{ }_{n} \geq 1\right.$ de $G$, tels que la suite de mesures de probabilité $\left\{U_{n}\left(\lambda^{n}\right)\right\}_{n \geq 1}$ converge vaguement vers une mesure de probabilité sur $G$ pas trop dégénérée.

Nous commençons (partie I) par étudier le cas d'un produit semi-direct d'un groupe de Lie nilpotent simplement connexe par un . groupe compact. Nous montrerons par la suite (partie II) que le cas général se ramène à ce cas particulier. 

1 Thêorème de la limite centrale pour un produit semi-direct d'un groupe de Lie nilnotent simplement connexe par un groupe compact.

Soit $G=N x_{n} K$ le produit semi-direct d'un groupe de Lie nilpotent simplement connexe $N$ par un groupe compact $K$ associé à un homomorphisme continu $n$ de $K$ dans le groupe des automorphismes de $N^{\prime}$. Le produit de deux éléments $g=(u, k)$ et $g^{\prime}=\left(u^{\prime}, k^{\prime}\right)$ de $G$ est défini par $g g^{\prime}=\left(u\left[n(k)\left(u^{\prime}\right)\right], k^{\prime}\right)$.

Soit $\lambda$ une mesure de probabilité sur les boréliens de G. Nous savons $([1])$ que $1 \mathrm{a} \mathrm{n}^{\text {ième }}$ convolée, $\lambda^{\mathrm{n}}$, de $\lambda$ converge raguement vers zéro. Nous nous proposons de trouver une suite d'homéomorphismes(si possible d'automorphismes) $\left\{\mathrm{I}_{n}\right\}_{n \geq 1}$ de $G$, tels que la suite de mesures de probabilité $\left\{V_{n}\left(\lambda^{n}\right)\right\}_{n \geq 1}$ converge vaguement vers une mesure de probabilité sur $G$, non dégénérée, en un sens à préciser.

\section{Analyse du Problème}

Nous désignons par $\pi_{1}$ et $\tau_{2}$ les projections de $G$ respectivement sur $N$ et $K$; nous notons $\lambda_{1}$ et $\lambda_{2}$ les images de $\lambda$ respectivement par $\pi_{1}$ et $\pi_{2}$.

(1.1) La projection $\pi 2^{\left(\lambda^{n}\right)}$ de $\lambda^{n}$ sur $k$ est la'nième convolée de $\lambda_{2}$. Désignons par $K\left(\lambda_{2}\right)$ (resp. $\hat{K}^{\pi}\left(\lambda_{2}\right)$ ) le sous-groupe compact 
de $K$ engendrê par le support $S\left(\lambda_{2}\right)$ de $\lambda_{2}$ (resp. par

$\left.S\left(\lambda_{2}\right)\left[S\left(\lambda_{2}\right)\right]^{-1}\right) \cdot \tilde{\kappa}\left(\lambda_{2}\right)$ est un sous-groupe distingué de $K\left(\lambda_{2}\right)$; nous disons que $\lambda_{2}$ est apériodique si $\tilde{K}\left(\lambda_{2}\right)=k\left(\lambda_{2}\right)$. Nous savons que, $([7])$, pour tout élément $k$ de $S\left(\lambda_{2}\right)$, la suite de mesures de probabilité $\left\{\varepsilon_{k_{n}-n} * \lambda_{2}^{n}\right\} n \geq 1$ converge vaguement vers la mesure

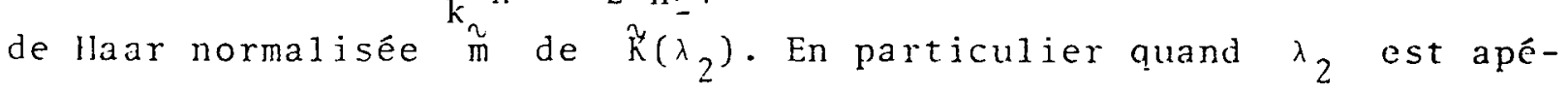
riodique, 1 a suite de mesures de probabilité $\left\{\lambda_{2}^{n}\right\}_{n \geq 1}$ converge vaguement vers la mesure de Har normalisée $\mathrm{m}$ de $\mathrm{k}\left(\lambda_{2}\right)$.

D'autre part, nous avons; avec les notations précédentes :

\section{Proposition.}

Soit $\left(x_{i}\right)_{i \geq 1}$ une suite de $v \cdot a$. indépendantes définies sur un espace probabilisé $(\Omega, a, P)$, à valeurs dans $G$, et de loi commune $\lambda$. Soit $\left\{U_{n}{ }_{n} \geq 1\right.$ une suite d'homéomorphismes de $N^{1}$ tels que : la suite demesures de probabilité sur $x\left\{U_{n} \circ \pi_{1}\left(\lambda^{n}\right)\right\}_{n \geq 1}$ converge en loi vers une mesure de probabilité $v$; et, pour tout entier naturel $p$, pour toute fonction $f$ continue, à support compact sur $N$,

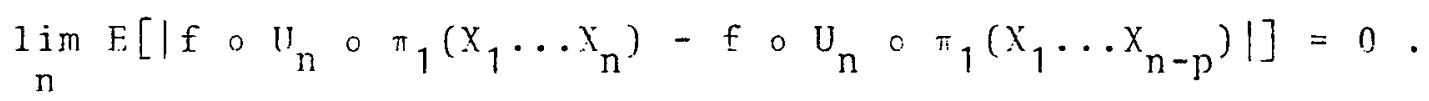

lour tout élément $k$ de $k$, et tout entier naturel $n$, posons $V_{n}^{k}(g)=\left(U_{n} \circ \pi_{1}(g), k^{-n_{\pi_{2}}}(g)\right)$.

Alors, pour tout élément $k$ de $S\left(\lambda_{2}\right)$, la suite de mesure de probabilité sur $G\left\{T_{n}^{k}\left(\lambda^{n}\right)\right\}_{n \geq 1}$ converge vaguement vers la mesure de probabilité produit $\nu \otimes \tilde{m}$. En particulier lorsque $\lambda_{2}$ est apériodique, $V_{n}^{e}\left(\lambda^{n}\right)$, (e désignant l'élément neutre de $k$ ), converge vaguement vers la mesure produit $v \otimes \mathrm{m}$.

De $(1.1)$ et $(1.2)$, il résulte que le problème considéré se ramène à trouver une suite d'homéomorphismes $\left\{U_{n}\right\}_{n \geq 1}$ de $x$ possì- 
dant les deux propriétés énoncées dans la proposition (1.2) et pour laquelle la limite vague $v$ de $U_{n} \circ \pi_{1}\left(\lambda^{n}\right)$ soit non dégénérée (i.e. non portée par une sous variété propre de N).

(1.3) Pour toute mesure de probabilité $\mu$ sur G, nous désignerons par $Q_{k}$ l'opérateur de transition sur $N$, identifié à $\mathrm{G} / \mathrm{K}$, associé à $\mu$; nous avons

$$
\begin{aligned}
r_{\mu} f(v) & =\int_{G} f(g \cdot x) \mu(d g) \\
& =\int_{N x K} f(u[n(k)(v)]) \mu(d u, d k) \quad(v \in N),
\end{aligned}
$$

pour toute fonction horélienne $f$ sur $N$ pour laquelle, le second membre est défini pour tout $u \in N$. Pour une mesure de Ilirac $\varepsilon_{g}$, $g \in G$, nous notons $Q_{g}$ au lieu de $Q_{\varepsilon}$. Si $\mu$ et $\mu$ ' sont deux mesures de probabilité sur $G$, nous avons $Q_{\mu} Q_{\mu}{ }^{\prime}=Q_{\mu} *^{*}$. En particulier si $\mu$ est une mesure de probabjlité sur $G$ idempotente, c'est-à-dire est la mesure de Haar normalisée d'un sous-groupe compact de $G$, alors $Q_{\mu}$ est un projecteur.

Avec ces notations, nous avons, pour toute fonction borélienne bornée (ou positive) sur $N$,

$$
\int_{N} f(u) \pi_{1}\left(\lambda^{n} * \varepsilon_{v}\right)(d u)=Q_{\lambda}^{n} f(v), \quad(v \in N) .
$$

Pour étudier la suite de mesures de probabilité ${ }^{\pi}{ }_{1}\left(\lambda^{n}\right)$, nous sommes ainsi amenés à étudier la suite d'opérateurs $\left\{\Omega_{\lambda}^{n}\right\}_{n \geq 1}$. Cette étude sera faite dans la section 2 dans une situation particulièrement favorable. Par la suite (section 5 ) nous montrerons que le cas général (moyennant des hypothèses de moment sur $\lambda$ ) se ramène toujours à cette situation particulière. Nous terminons cette section 1 en donnant une démonstration de la proposition (1.2) 
Preuve de la proposition (1.2)

Nous commençons par établir un lemme.

\section{(1.4) Lemme :}

Soit $\left\{\mu_{n}\right\}_{n \geq 1}$ une suite de mesures de probabilité sur un groupe compact $K$, convergeant vaguement vers une mesure de probabilité $\mu$. Alors pour toute fonction $f$ continue sur $k$, nous avons

$$
\operatorname{Iim} \sup _{x \in K}\left|\int_{K} f(x k) \mu_{n}(d k)-\int_{K} f(x k) \mu(d k)\right|=0 .
$$

Preuve du lemme (1.4):

Si $h$ est une fonction continue sur $K$, nous posons ||$h \|_{\infty}=\sup _{x \in K}|h(x)|$ et nous notons $h^{x}$, la translaté à gauche de $h$ parl'élément $x$ de $k\left(i . e . ~ h^{x}(k)=h(x k)\right.$, th $\left.\epsilon^{k}\right)$.

Soit $f$ une fonction continue sur $k$. Pour tout entier naturel non nul $m$, posons

$$
f_{m}(x)=\sup _{n \geq m}\left|\int_{K} f(x k) \mu_{n}(d k)-\int_{K} f(x k) \mu(d k)\right|,(x \in K) .
$$

On vêrifie aisément que l'on a :

$$
f_{m}(x) \leq f_{m}(y)+2\left\|f^{x y^{-1}}-f\right\|_{\infty}, F(x, y) \in k x k ;
$$

d'où

$$
\begin{aligned}
& \left|f_{m}(x)-f_{m}(y)\right| \leq 2 \sup \left\{|| f^{x y^{-1}}-f||_{\infty},|| f y x^{-1}-f||_{\infty}\right\}, \\
& \mathrm{H}(\mathrm{x}, \mathrm{y}) \text { e } \mathrm{KxK} \text {; }
\end{aligned}
$$

et par suite, pour tout entier $m \geq 1$, la fonction $f_{m}$ est continue sur $\mathrm{K}$.

Soit $\varepsilon$ un réel $>0 .\left(\left\{f_{m}<\varepsilon\right\}\right)_{m \geq 1}$ est une famille croissante d'ouverts de $K$. Comme la suite $\left\{\mu_{n}\right\}_{n \geq 1}$ converge vaguement vers $\mu$, cette famille constitue un recouvrement ouver't de $K$. Ne 
la compacité de $k$, il résulte alors qu'il existe un entier $m(\varepsilon)$ pour lequel $K=\left\{f_{m(\varepsilon)}<\varepsilon\right\}$. Le lemme est prouvé.

La proposition (1.2) résulte alors du lemme suivant.

\section{(1.5) Lemme :}

Soit $\left(X_{i}\right)_{i \geq 1}$ une suite de v.a. indépendantes définies sur un espace probabilisé $(\Omega, a, \mathbb{P})$, à valeurs dans $G$, et de loi commune $i$; pour tout entier naturel non nul $n$, nous posons $s_{n}=x_{1} \ldots x_{n}$.

Soit $\left\{U_{n}\right\}_{n \geq 1}$ une suite d'homéomorphismes de $N$ tels que, pour tout entier naturel $p$, pour toute fonction $f$ continue, à support compact sur $N$,

$$
\lim _{n} E\left[\left|f \circ U_{n} \circ \pi_{1}\left(s_{n}\right)-f \circ u_{n} \circ \pi_{1}\left(s_{n-p}\right)\right|\right]=0 \text {. }
$$

Alors les v.a. $U_{n} \circ \pi_{1}\left(S_{n}\right)$ et $\pi_{2}\left(S_{n}\right)$ sont asymptotiquement indépendantes ; c'est-à-dire que pour toute fonction continue $f$, à support compact, sur $N$ et pour toute fonction $h$ continue sur $K$, nous avons

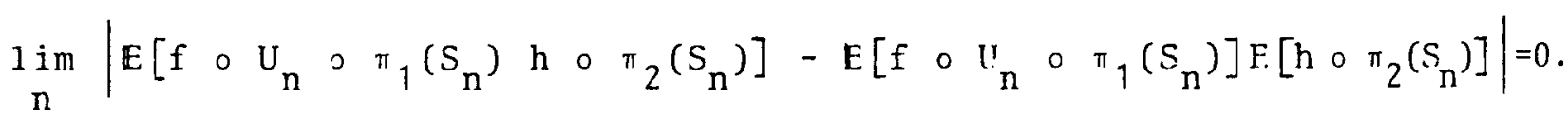

Preuve du lemme $(1.5)$ :

Pour tout entier naturel $p$, nous avons

$$
\begin{aligned}
& \mid \mathbb{E}\left[\begin{array}{llllllllll}
f & \circ & u_{n} & \circ & \pi_{1}\left(S_{n}\right) & h & \circ & \pi_{2}\left(S_{n}\right)
\end{array}\right]-E\left[\begin{array}{llllll}
f & \circ & u_{n} & \circ & \pi_{1}\left(S_{n}\right)
\end{array}\right] E\left[\begin{array}{lll}
h & \circ & \pi_{2}\left(S_{n}\right)
\end{array} \mid\right. \\
& \leq \alpha(n, p)+B(n, p) .
\end{aligned}
$$

avec, 


$$
\begin{aligned}
& a(n, p)=\left|F\left[\left(f \circ U_{n} \circ \pi_{1}\left(S_{n}\right)-f \circ U_{n} \circ \pi_{1}\left(S_{n-p}\right)\right) h \circ \pi_{2}\left(S_{n}\right)\right]\right| \\
& \leq|| h||_{\infty} E\left[\left|f \circ u_{n} \circ \pi_{1}\left(S_{n}\right)-f \circ u_{n} \circ \pi_{j}\left(S_{n-p}\right)\right|\right]
\end{aligned}
$$

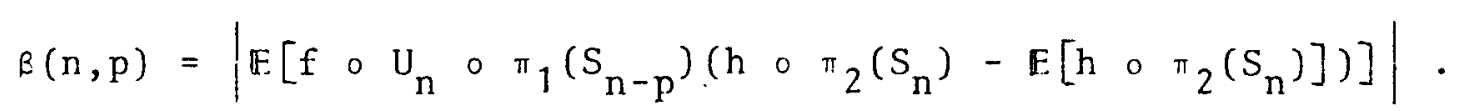

Comme les v.a. $\left(x_{i}\right)_{i \geq 1}$ sont indépendantes et de loi $\lambda$, nous avons

$B(n, p) \leq|| f||_{\infty}\left|E\left[\int_{K} h\left(\pi_{2}\left(S_{n-p}\right) k\right) \lambda \frac{p}{2}(d k)\right]-\int_{K} h(k) \lambda_{2}^{n}(d k)\right|$.

Soit $k_{0}$ un élément de $S\left(\lambda_{2}\right) \cdot k_{0}^{-n} s_{n-p} k_{0}^{p} s^{\prime e ́ c r i t ~}$

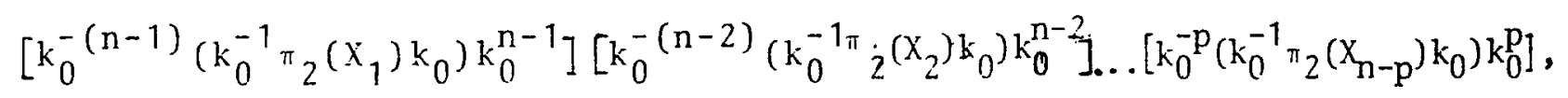
et appartient donc, $\mathbb{P}-$ p.s., à $\mathbb{k}^{x}\left(\lambda_{2}\right)$. On en déduit que $\beta(n, p) \leq|| f||_{\infty} \sup _{x \in K^{k}\left(\lambda_{2}\right)}\left|\int_{K} h\left(k_{0}^{n} x k\right) \mu_{p}(d k)-\int K_{K} h\left(k_{0}^{n} k\right) \mu_{n}(d k)\right|$, où $\left\{\mu_{n}\right\}_{n \geq 1}$ désigne la suite de mesure de probabilité $\left\{\varepsilon_{k}-n{ }_{0}^{\left.* \lambda_{2}\right\}} n \geq 1\right.$

Comme la suite $\left\{\mu_{n}\right\}_{n \geq 1}$ converge vaguement vers $\tilde{m}$, du lemme (1.4) il résulte que $l$ 'on a $\lim \sup B(n, p)=0$.

$$
\mathrm{p} n \geq \mathrm{p}
$$

D'autre part, I'hypothèse du lemme (1.5), nous assure que pour tout entier $p$ fixé $\lim \alpha(n, p)=0$.

Le lemme (1.5) est prouvé. 


\section{2. "Résolution" du Problème dans une situation particulière}

(2.1) Nous pouvons toujours supposer, qu'en tant qu'espace topologique, $N$ est un espace vectoriel réel de dimension finie et que pour tout élément $k$ de $K, n(k)$ est un automorphisme d'espace vectoriel de $\mathbf{N}$. [En effet, si ce n'est pas le cas, munissons 1'algébre de lie $(\mathscr{L}(N),[]$,$) du produit o défini par la formule$ de Campbe11-Hausdorff,

$$
u \circ v=u+v+\frac{1}{2}[u, v]+\ldots \quad(u, v \in N)
$$

nous savons que l'application exponentielle de $N$ est un isomorphisme de groupe analytique de $(\mathscr{L}(\mathbb{N}), 0)$ sur $N ;$ le groupe $G$ est alors isomorphe au produit semi-direct $(\mathcal{L}(N), 0) x_{\mathscr{L}(n)} K$, où pour tout $k \in K$, on désigne par $\mathscr{L}(n)(k) 1$ 'automorphisme de $(\mathcal{L}(N),[]$, et par suite de $(\mathscr{L}(N), 0)$, tangent à l'automorphisme $n(k)$ de $N]$.

D'autre part, quitte à remplacer $G=N x_{n} K$ par $N x_{n} K\left(\lambda_{2}\right)$, nous pouvons supposer que $K\left(\lambda_{2}\right)=K$.

Nous faisons alors sur le couple $(G, \lambda)$ l'hypothèse suivante : Il existe sur l'algèbre $\mathbf{C}[\mathrm{N}]$ des fonctions polynomes sur $N$, à coefficients complexes, une notion de degré pour laquelle

i) VT $\varepsilon \in \mathbb{C}[N], T(u v)=T(u)+T(v)+P_{T}(u, v),(u, v \in N)$,

où $\mathrm{P}_{\mathrm{T}}$ est une fonction polynome sur $N \times N$ dont le degré global, $\mathrm{dg} \mathrm{P}_{\mathrm{T}}$, est inférieur ou égal à celui, $\mathrm{dgT}$, de $\mathrm{T}$; et les valuations partielles de $P$, val $P / u$ et val $P / v$, sont supérieurés ou égales à 1 
ii) $\forall \mathrm{FT} \mathbb{C}[\mathrm{N}], \operatorname{dgT} \circ \eta(\mathrm{k})=\mathrm{dgT}, \forall \mathrm{Fk} \mathrm{K}$.

iii) Pour toute forme linéaire $A$ sur $N$ de degré 1 ,

$$
\int_{N} \sup _{k \in K}|A(n(k)(u))| \lambda_{1}(d u)<+\infty
$$

et

$$
\int_{N} \int_{K} A(n(k)(u)) m(d k) \lambda_{1}(d u)=0
$$

(2.2) Remarque. Une notion de degré sur $\mathbb{C}[N]$, s'obtient obligatoirement en choississant une base. $\left\{f_{i}\right\}_{1 \leq i \leq p}$ de 1 'espace vectoriel $N$, en attribuant un degré à chaque fonction coordónnée $y_{i}, 1 \leq i \leq p$, associée à cette base et en convenant que le degré (resp. la valuation) du polynome nul est $(-\infty)$ (resp. $(+\infty))$. Nous disons alors que $\left\{f_{i}\right\}_{1 \leq i \leq p}$ est une base de référence pour cette notion de degré.

Pour qu'une notion de degré vérifie l'hypothèse i) resp ii)) de (2.1) il faut et il suffit que les relations de i) (resp. ii)) soient vérifiées par les fonctions coordonnées $\left(y_{i}\right)_{1 \leq i \leq p}$ d'une de ses bases de référence. Dans ce cas, on remarquera que l'élément neutre et 1 'inverse d'un élément $u$ du groupe $N$ coincident respectivement avec l'élément nul et l'opposé de u dans le groupe additif de l'espace vectoriel $N$. En outre, pour toute forme linéaire A de degré 1 sur $N$ (i.e. pour toute combinaison 1 inéaire des fonctions coordonnées $y_{i}$ de degré 1), nous avons $A(u v)=A(u)+A(v)=A(u+v)$.

Pour tout élément $u$ de $N$, désignons par $L_{u}$ (resp. $F_{u}$ ) la multiplication à gauche (resp. à droite) par u sur $N$. Pour tous éléments $g=(u, k)$ de $G$ et $T$ de $C[N]$, nous avons, (voir (1.4)),

$$
\left.Q_{g} T=T \circ I_{u} \circ n(k)\right)
$$


D'après les hypothèses ii) et iii) de (2.1), on voit qu'ainsi G opère à droite sur $C[N]$ et les sous-espaces $\mathbb{C}_{\ell}[N]=\{\mathrm{T} \in \mathbb{C}[]:. \mathrm{dgT} \leq \ell\}, \ell \in \mathbb{N} ;$ en outre, nous atons

$$
\left(Q_{g}-Q_{k}\right) T(\cdot)=P_{T}(u, n(k)(\cdot))+T(u),
$$

qui montre que

$$
\operatorname{dg}\left(Q_{g}-Q_{k}\right) T \leq d g T-1, \forall g=(u, k) \quad c \quad G, V T \in C[N]
$$

Soit $\mu$ une mesure de probabilité sur G . Posons

$\ell(\mu)=\sup \left\{\ell \in \mathbb{N}: \int_{N} \sup _{k \in K}|T \circ n(k)(u)| \pi_{T}(\mu)(d u) \cdot+\infty, \forall T \in \mathbb{C}_{\ell}[N]\right\}$.

l)e ce qui précède, il résulte que $Q_{\mu}$ opère sur $\mathbb{C}_{\ell(\mu)}[\bar{N}]$, (on convicnt que $\mathbb{C}_{+\infty}[N]=\mathbb{C}[N] !$ ), et nous avons :

\section{(2.4) Lemme}

Soit $\mu$ une mesure de probabilité sur G. Alors pour tout $T \in \mathbb{C}_{\ell(\mu)}[\mathrm{N}]$,

$$
\operatorname{dg}\left(Q_{\mu}-Q_{\pi_{2}(\mu)}\right) T \leq \operatorname{dgT}-1 .
$$

Si de plus, $\mu$ vérifie $\int_{N} A(u) \pi_{1}(\mu)(d u)=0$, pour toute forme linéaire $A$ de degré 1 sur $N$, alors

$$
\operatorname{dg}\left(Q_{\mu}-Q_{2}(\mu)\right)^{T} \leq \mathrm{dg} T-2
$$

(2.5) L'opérateur $Q_{\lambda_{2}}\left(I-Q_{m}\right)=\left(1-Q_{m}\right) Q_{\lambda_{2}}$ de $\mathbb{C}[N]$ ne possède pas la valeur propre 1. Ln effet, si $T \in \mathbb{C}[N]$ vérifie $Q_{\lambda_{2}}\left(I-Q_{m}\right) T=T$, nous avons 


$$
T=\frac{1}{n}\left(\sum_{k=1}^{n} Q_{\lambda}{ }_{2}\right)\left(I-Q_{m}\right) T
$$

et par suite, puisque la suite de mesures de probabilité $\left\{\frac{\lambda_{2}+\ldots+\lambda_{2}^{n}}{n}\right\} \underset{n \geq 1}{n}$ converge vaguement vers $m, T=Q_{m}\left(I-Q_{m}\right) T=0$. I1 s'ensuit que $\left(I-Q_{\lambda_{2}}\left(I-Q_{m}\right)\right)$ est un opérateur bijectif de $\mathbf{C}[N]$.

Désignons par $\bar{\lambda}$ la mesure de probabilité sur $\mathrm{N}$ définie par $\int_{N} n(k)\left(\lambda_{1}\right) m(d k)$. Appelons $\hat{\lambda}$ la mesure de probabilité sur $N$ définie par

$$
\hat{\lambda}(f)=\int_{N x K} f\left(n\left(k^{-1}\right)(u)\right) \lambda(d u, d k),
$$

pour toute fonction borélienne bornée $f$ sur $N$.

Posons

$$
\begin{aligned}
L_{\lambda} & =Q_{m}\left(Q_{\lambda}-I\right) Q_{m}+Q_{m}\left(Q_{\lambda}-I\right)\left[I-Q_{\lambda_{2}}\left(I-Q_{m}\right)\right]^{-1}\left(Q_{\lambda}-I\right) Q_{m} \\
& =Q_{m}\left(Q_{\bar{\lambda}}-I\right) Q_{m}+Q_{m}\left(Q_{\lambda_{1}}-I\right)\left[I-Q_{\lambda_{2}}\left(I-Q_{m}\right)\right]^{-1}\left(Q_{\lambda}:-I\right) .
\end{aligned}
$$

D'après le lemme (2.4), l'opérateur $L_{\lambda}$ baisse le degré de tout élément de $\mathbb{C}_{\ell(\lambda)}[N]$ d'au moins deux unités.

Nous avons alors:

(2.6) Proposition :

Soient $G=N x_{n} K$ un produit semi-direct d'un groupe de Lie nilpotent simplement connexe par un groupe compact $K$ et $\lambda$ une mesure de probabilité sur $G$. Supposons que le couple $(G, \lambda)$ vérifie les hypothèses de $(2.1)$. 
Alors, (avec les notations de (2.5)), pour toute fonction polynome $T$ sur $N$ de degré $\leq \ell(\lambda)$, la suite de fonctions polynomes $\left\{\frac{1}{n} \frac{1}{d g T / 2} Q_{\lambda}^{n} T\right\}{ }_{n \geq 1}$ converge, uniformément sur tout compact de $N$, vers zéro si dgT est impair, vers la constante $\frac{1}{(\operatorname{dgT} / 2) !} L_{\lambda}^{\operatorname{dgT} / 2} \mathrm{~T}$ si dgT est pair.

Une démonstration de la proposition (2.6) sera donnée dans la section 3 . On remarquera que cette proposition améliore un résultat de Guivarc'h ([2] corollaire de la proposition 2).

Nous allons à présent donner deux corollaires.

\section{(2.7) Coroliaire.}

Soit $(G, \lambda)$ comme dans la proposition (2.6). Supposons que $\lambda$ vérifie, en outre, 1 'une des deux propriétés:

$$
\lambda=\lambda_{1} \otimes \lambda_{2} \text { ou } \int A(u) \lambda_{1}(d u)=0 \text {, pour toute forme Iinéaire }
$$

A de degré 1 sur $N$.

Alors, pour tout élément $T$ de $\mathbf{C}_{\ell(\lambda)}[N]$ de degré pair, nous avons

$$
\mathrm{L}_{\lambda}^{\mathrm{dgT} / 2} \mathrm{~T}=\left(\mathrm{Q}_{\bar{\lambda}}-\mathrm{I}\right)^{\mathrm{dgT} / 2} \mathrm{~T}
$$

Preuve.

$$
\text { Si } \lambda=\lambda_{1} \otimes \lambda_{2} \text {, nous avons }\left(Q_{\lambda}-I\right) Q_{m}=Q_{\lambda_{2}}\left(Q_{\bar{\lambda}}-I\right) Q_{m} \text {. }
$$

Par suite, dans les deux cas envisagés dans le corollaire, l'opérateur

$$
Q_{m}\left(Q_{\lambda_{1}}-I\right)\left[I-Q_{\lambda_{2}}\left(I-Q_{m}\right)\right]^{-1}\left(Q_{\lambda}-I\right) Q_{m}
$$

baisse le degré de toute fonction polynome de degré $\leq \ell(\lambda)$ d'au moins 
trois unitês. Nous avons alors

$$
\mathrm{L}_{\lambda}^{\mathrm{dgT} / 2} \mathrm{~T}=\left[\mathrm{Q}_{\mathrm{m}}\left(\mathrm{Q}_{\bar{\lambda}}-\mathrm{I}\right) \mathrm{Q}_{\mathrm{m}}\right]^{\mathrm{dgT} / 2} \mathrm{~T}=\left(\mathrm{Q}_{\bar{\lambda}}-\mathrm{I}\right)^{\mathrm{dgT} / 2} \mathrm{~T} .
$$

\section{(2.8) Corollaire.}

Soit $(G, \lambda)$ comme dans la proposition $(2.6)$. Soit a un élément de $N$ vérifiant

$$
A(a)=\left(\left[I-Q_{\lambda_{2}}\left(I-Q_{\dot{m}}\right)\right]^{-1} A\right)\left(\int_{N} u \lambda_{1}(d u)\right),
$$

pour toute forme linéaire $A$ sur $N$ de degré 1.

$$
\text { Alors, pour tout élément } T \text { de } \mathbb{C}_{\ell(\lambda)}[\mathrm{N}] \text { de degré pair, }
$$

nous avons

$$
\mathrm{L}_{\lambda}^{\mathrm{dg} T / 2} \mathrm{~T}=\left(\mathrm{Q}_{\overline{\lambda(\mathrm{a})}}-\mathrm{I}\right)^{\mathrm{dgT} / 2 \mathrm{~T}},
$$

où $\lambda(a)=\varepsilon(-a, e) * \lambda * \varepsilon(a, e)$ et $\overline{\lambda(a)}=\int_{K} n(k) \circ \pi_{1}(\lambda(a)) m(d k)$.

Preuve.

Pour tout élément a de $N$ vérifiant la relation (*), on voit facilement que l'on a

$$
\int_{N} A(u) \pi_{1}(\varepsilon(-a, e) * \lambda * \varepsilon(a, e))(d u)=0 \text {, pour }
$$

toute forme linéaire A de degré 1 sur $N$. Autrement dit la mesure de probabilité sur $G, \lambda(a)$, vérifie l'hypothèse du corollaire (2.7).

$$
\begin{aligned}
& \text { Or pour tout entier naturel } n \geq 1 \text {, nous avons } \\
& Q_{\lambda}^{n}=Q_{(a, e)}^{-1} Q_{\lambda(a)}^{n} Q_{(a, e)}
\end{aligned}
$$


On en déduit que

$\left.\lim \frac{1}{n^{d g T / 2}} Q_{\lambda}^{n} T=\frac{1}{\left(\frac{d g T}{2}\right) !}\left(Q_{\lambda(a)}-I\right)^{d g T / 2} Q_{(a, e)}\right)^{T}=\frac{1}{\left(\frac{d g T}{2}\right) !}\left(Q_{\overline{\lambda(a)}}-I\right) d g T / 2 T$

car $\operatorname{dg}\left(Q_{(a, e)}-I\right) T \leq d g T-1 ;$ d'où le corollaire.

(2.9) Choisissons une base $b=\left\{e_{i}\right\}_{1 \leq i \leq p}$ de l'espace vectoriel $\mathrm{N}$ qui soit une base de référence pour la notion de degré sur $\mathrm{C}[\mathrm{N}]$ considérée en (2.1), (voir remarque (2.2)). Notons $\left\{x_{i}\right\}_{1 \leq i \leq p}$ le système de fonctions coordonnées associé à cette base. Pour tout réel $t>0$, nous posons

$$
u_{t}^{b}(u)=\sum_{i=1}^{p} \frac{x_{i}(u)}{t^{d g x_{i} / 2}} e_{i} \quad(u \in N) \quad ;
$$

puis

$$
\mathrm{U}_{t}^{\mathrm{b}}(\mathrm{u}, \mathrm{k})=\left(\mathrm{U}_{\mathrm{t}}^{\mathrm{b}}(\mathrm{u}), \mathrm{k}\right) \quad, \quad((\mathrm{u}, \mathrm{k}) \in \mathrm{N} \times \mathrm{K})
$$

(2.10) Soient $u$ et $v$ deux éléments de $N$. Pour tout i $\in\{1, \ldots, p\}$, écrivons, (hypothèse ii) de (2.1)),

$$
x_{i}(u v)=x_{i}(u)+x_{i}(v)+P_{i}(u, v),
$$

où $P_{i} \in \mathbb{C}\left[\begin{array}{lll}N & x & N\end{array}\right]$ tel que dg $P_{i} \leq d g x_{i}$, valp $P_{i} / u$ et $\operatorname{val} P_{i} / v \geq 1$.

Alors, pour tout i $\in\{1, \ldots, p\}$, nous avons

$x_{i}\left(U_{t}\left(U_{1 / t}(u) U_{1 / t}(v)\right)\right)=x_{i}(u)+x_{i}(v)+\frac{1}{t} \frac{d g x_{i} / 2}{P_{i}}\left(U_{1 / t}(u), U_{1 / t}(v)\right)$ 
ct par suite,

$\lim _{t \rightarrow+\infty} x_{i}\left(U_{t}\left(U_{1 / t}(u) U_{1 / t}(v)\right)\right)=x_{i}(u)+x_{i}(v)+\overline{p_{i}}(u, v)$,

où $\bar{P}_{i}$ est le polynome sur $N \times N$ qui se déduit de $P_{i}$ en conservant uniquement les monomes de degré $d g x_{i}$.

En posant

$$
u \cdot v=\lim _{t \rightarrow+\infty} U_{t}\left(U_{1 / t}(u) U_{1 / t}(v)\right)
$$

on vérifie facilement que l'on définit un produit nilpotent sur l'espace vectoriel $N$; nous notons $N^{b}$ le groupe de Lie nilpotent simplement connexe $(N, \cdot)$. Pour tout $t>0, u_{t}^{b}$ est un automorphisme de $\mathrm{N}^{\mathrm{b}}$.

(2.11) Pour tout élément $g=(u, k)$ de $G$, nous posons

$$
\begin{aligned}
& \phi_{b}(g)=\phi_{b}(u)=\sup _{1 \leq i \leq p} \sup _{k \in K} \mid x_{i}\left(n(k)(u):\left.\right|^{1 / d g x_{i}}\right. \\
& \text { La condition }(*) \int_{G} \phi_{b}^{2}(g) \lambda(d g)<+\infty,
\end{aligned}
$$

est équivalente à

$$
\int_{N} \sup _{k \in K^{\prime}}|T(n(k)(u))|^{2 / d g T} \lambda_{1}(d u)<+\infty, V T \in C[N]
$$

et est par conséquent indépendante du choix de b.

$$
\text { Nous avons alors: }
$$

\section{(2.12) Théorème :}

Soit $(G, \lambda)$ vérifiant les hypothèses de (2.1). Supposons qu'en outre $\lambda$ vérifie la condition $(*)$ de (2.11). Désignons par $b=\left\{e_{i}\right\}_{1 \leq i \leq p}$ une base de référence pour 1 a notion de degré sur $\mathbb{C}[N]$ considérée en (2.1) et par $\left\{x_{i}\right\}_{1 \leq i \leq p}$ le système de fonctions 
coordonnćes associé à cette base.

Alors, (avec les notations de $(2.9)$ et $(2.10)$ ), la suite de mesures de probabilité $\quad\left\{U_{n}^{b} \circ \pi_{1}\left(\lambda^{n}\right){ }_{n} \geq 1\right.$ converge vaguenent vers la loi au temps 1 du semi-groupe de convolution $\left\{v_{t}^{3}\right\} t>0$ sur $x^{b}$ de génêrateur infinitésimal
$A=\left\{i: \operatorname{dg}^{\sum} x_{i}=2\right\}$
$\left(L_{\lambda} x_{i}\right) D_{i}+\frac{1}{2}$
$\left\{i, j: d g x_{i}=d g x_{j}=1\right\}$
$\left(\begin{array}{llllll}L_{\lambda} & x_{i} & x_{j}\end{array}\right) D_{i} D_{j}$

où, pour toute fonction $f$ de classe $C^{1}$ sur $N$,

$$
D_{i} f(u)=\lim _{t \rightarrow 0} \frac{f\left(u \cdot t e_{i}\right)-f(u)}{t}, u \in N \text {, i } \in\{1, \ldots, p\}
$$

En outre la suite d'homéomorphismes $\left\{U_{n}^{b}\right\}_{n} \geq 1$ vérifie les hypothèses de la proposition (1.2) ; les conclusions de cette proposition s'appliquent donc.

Une démonstration du théorème (2.12) sera donnée dans $1 \mathrm{a}$ section 4 . Nous terminons cette section en faisant quelques commentaires.

(2.13) Remarque. Pour savoir dans quels cas nous avons effectivement résolu le problème posé, nous devons étudier le semi-groupe $\left\{\nu_{t}\right\}_{t>0}$ de g.i. A.

Chaque- $\mathrm{D}_{i}, 1 \leq i \leq p$, est un champ analytione de vecteurs tangents, invariant à gauche sur $\mathrm{N}^{\mathrm{b}}$; c'est-à-dire un élément de l'algèbre de Lie, $\mathcal{L}\left(N^{b}\right)$, de $N^{b}$. 
Si pour tout $i \in\{1, \ldots, p\}, d g x_{i} \geq 2$, A est un élément de $\not{x}\left(N^{b}\right)$. Nous avons alors $\nu_{t}=\varepsilon_{t f_{0}}$, où $f_{0}=\left\{i: d g x_{i}=2\right\}\left(L_{\lambda} x_{i}\right) e_{i} \in N$; et par suite $U_{n}^{b} \circ \tau_{1}\left(\lambda^{n}\right)$ converge vaguement vers la mesure de Dirac ${ }^{\varepsilon} f_{0}$.

S'il existe des coordonnées $x_{i}$ de degré 1 , álors $A$ s'écrit sous la forme

$$
A=z_{0}+\frac{1}{2} \sum_{i=1}^{1} z_{i}^{2} \quad \text { avec } \quad s \in \mathbb{N}^{*}
$$

où $z_{i}, 0 \leq i \leq s$, sont des éléments de $\mathscr{L}\left(N^{b}\right)$.

Désignons par $\mathscr{L}_{1}(\mathrm{~A})$ la sous-algèbre de Lie de $\mathcal{L}\left(\mathrm{N}^{\mathrm{b}}\right)$ engendrée par les éléments $Z_{0}, \ldots, z_{s}$; et $\operatorname{par} \mathscr{L}_{2}(A)$ l'idéal de $\mathcal{L}_{1}$ (A) formé par les éléments de $\mathscr{L}_{1}(\mathrm{~A})$ de 1 a forme

$$
\sum_{i=1}^{S} \lambda_{i} Z_{i}+Y
$$

avec $\lambda_{i} \in R, 1 \leq i \leq r \quad$ et $\quad Y \in\left[\mathcal{L}_{1}(A), \mathscr{L}_{1}(A)\right]$.

En omettant 1'indice $A$, désignons par $L_{1}$ et $L_{2}$ les sous-groupes de Lie connexes de $N^{b}$ possèdant respectivement $\mathscr{L}_{1}$ et $\mathscr{Z}_{2}$ pour algèbre de Lie. $\mathrm{L}_{2}$ est un sous-groupe distingué de $L_{1}$.

Nous avons alors (voir $[4])$ :

1) si $\mathscr{L}_{1}=\mathscr{L}_{2}$ (i.e. $L_{1}=L_{2}$ ), le semi-groupe de convolution $v_{t}$ de g.i. A est absolument continu par rapport à la mesure de Haar de $L_{1}$, avec une densité de classe $C^{\infty}$.

2) si $\mathcal{L}_{1} \neq \mathcal{L}_{2}$, nous avons $\mathscr{L}_{1}=\mathscr{L}_{2} \oplus \mathbb{R} z_{0}$ et par suite, puisque $N^{b}$ est nilpotent simplement connexe, $L_{1}$ est un produit 
semi-direct de $L_{2}$ par $(\mathbb{R},+)$. Le semi-groupe de conrolution "t de g.i. A s'écrit alors $v_{t}=H_{t} \otimes e_{t}$, où $H_{t}$ est absolument continue par rapport à la mesure de Haar de $L_{2}$ arec une densité de classe $C^{\infty}$.

Posons $I=\left\{i \in\{1, \ldots, p\}: d g x_{i}=1\right\}$. Dans $1 \mathrm{c}$ cas où $1 \mathrm{a}$ matrice carrée $\left(\left(L_{\lambda} x_{i} x_{j}\right)_{i, j E I}\right.$ est définie positive, la sousalgèbre de Lie de $\mathcal{L}\left(N^{b}\right)$ engendrée par $z_{1}, \ldots, z_{s}$ est égale à celle engendrée par les $D_{i}, i \in I . L_{1}(A)$ est alors la sous-algèbre de $\mathscr{L}\left(\Lambda^{b}\right)$ engendrée par $z_{0}$ et les $D_{i}$, i $\varepsilon$ I $\mathscr{L}_{2}(A)$ est l'idéal de $\mathscr{L}_{1}(A)$ formé par les éléments de $\mathscr{L}_{1}$ de la forme

$$
\sum_{i \in I} \lambda_{i} D_{i}+Y \quad, \quad \lambda_{i} \in \mathbb{R} \text { et } \quad Y \in\left[\alpha_{1}^{*}, \mathscr{L}_{1}\right] .
$$

\section{(2.14) Lemme.}

Soit $(G, \lambda)$ vérifiant les hypothèses de (2.1). Supposons en outre que $\ell(\lambda) \geq 2$. Désignons par $G_{\lambda}$ le sous-groupe fermé de $G$ engendré par le support de $\lambda$ et par a un élément de $N$ vérifiant la relation (*) du corollaire (2.8).

Alors si la matrice $\left(\left(L_{\lambda} x_{i} x_{j}\right)_{i, j \in I} n^{\prime e s t}\right.$ pas définie positive, nous avons, (en identifiant $N$ et $K$ à des sous-groupes de G)

$$
\mathrm{G}_{\lambda} \subset \mathrm{N}_{0}\left(\mathrm{aka} \mathrm{a}^{-1}\right)
$$

où $\quad \Sigma_{0}$ est d'une part un sous-groupe fermé propre de $N$, distingué dans $G$, contenant le groupe dérivé de $N$, et d'autre part un sous-espace vectoriel propre de $\mathrm{N}$.

Preuve: D'après le corollaire (2.8), la matrice considérée n'est 
pas définie positive si et seulement s'il existe une forme linéaire non nulle $A$ sur $N$, de degré 1, telle que :

$$
\begin{aligned}
& \text { pour } \lambda(a) \text {-presque tout élément } g \text { de } G, A \circ \pi_{1}(k g)=0 \\
& \text { Yk } \in \mathrm{K}
\end{aligned}
$$

$$
\text { Soit } N_{0}=\left\{u \in N: A \circ \pi_{1}(k u)=0 \quad r k \in K\right\} \cdot N_{0} \text { est }
$$

un sous-groupe propre de $N$, distingué dans $G$ et contenant le groupe dérivé de $N^{\prime}$; en outre $N_{0}$ est un sous-espace vectoriel propre de $N$.

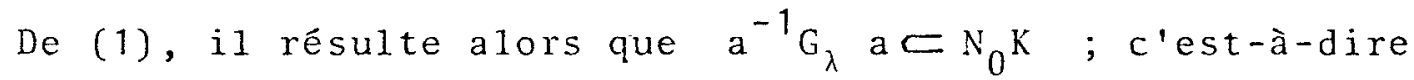
$\mathrm{G}_{\lambda} \subset \mathrm{N}_{0}\left(\mathrm{a} \mathrm{K} \mathrm{a}{ }^{-1}\right)$.

(2.14) Pour $k \in K$ et $u \in N$, définissons

$$
n^{b}(k)(u)=\lim _{t \rightarrow+\infty} U_{t}\left(n(k)\left(U_{1 / t}(u)\right)\right)
$$

On voit facilement que cette limite existe et que 1 'on a, $n^{b}(k)\left(e_{i}\right)=\left\{j ; \sum x_{j}=d g x_{i}\right\} x_{j}\left(n(k)\left(e_{i}\right)\right) e_{j}, \forall i \in\{1, \ldots, p\}$.

' D'où l'on déduit que $n^{b}$ est un homomorphisme continu de $K$ dans le groupe des automorphismes de $N^{b}$.

Nous notons $G^{b}=N^{b} x_{n} b$ le produit semi-direct du groupe de Lie nilpotent simplement connexe $\mathrm{N}^{\mathrm{b}}$ par le groupe compact $\mathrm{K}$ associé à $n^{b}$. Le produit - de deux éléments $(u, k)$ et $(v, h)$ de $G^{b}$ est défini par

$$
(u, k) \cdot(v, h)=\lim _{t \rightarrow+\infty} U_{t}\left(U_{1 / t}(u, k) U_{1 / t}(v, h)\right) .
$$

Pour tout $t>0, U_{t}^{b}$ est un automorphisme de $G^{b}$. Pour toute mesure de probabilité $\mu$ sur $N \times K$, désignons par $Q_{\mu}^{b}$ 1'opérateur de transition sur $\mathrm{N}^{\mathrm{b}}$, identifié $\mathrm{a} \mathrm{G}^{\mathrm{b}} / \mathrm{K}$, 
associề à $\mu$ (voir $(1 . \overline{3}))$.

Alors, nous avons :

(2.15) Lemme:

Soit $\left(G=N x_{\eta} k, \lambda\right)$ vérifiant les hypothèses de (2.1). Choisissons une base $b$ de $N$ et notons $G^{b}=N^{b}{ }_{n}{ }_{n} K$ le groupe associé à cette base par le procédé ci-dessus.

Alors, pour toute fonction polynome $T$ sur $N$ de degré $\leq \ell(\lambda)$, les limites des fonctions polynomes $\frac{1}{n^{d g T / 2}} Q_{\lambda}^{n} T$ et $\frac{1}{\mathrm{n} \operatorname{dgT/2}}\left(Q_{\lambda}^{\mathrm{b}}\right)^{\mathrm{n}} \mathrm{T}$ sont les mêmes.

Preuve : Soit $\mu$ une mesure de probabilité sur $N$. D'après (2.10), on voit facilement que, pour tout élément $\mathrm{T}$ de $\mathbf{C}_{\ell(\mu)}[\mathrm{N}]$,

$$
\operatorname{dg}\left(Q_{\mu}^{b}-Q_{\mu}\right) T \leq \operatorname{dg} T-2
$$

et même

$$
\operatorname{dg}\left(Q_{\mu}^{b}-Q_{\mu}\right) T \leq d g T-3
$$

si $\int_{N} A(u) \mu(d u)=0$, pour toute forme linéaire $A$ sur $N$ de degré $1 \cdot$.

D'autre part, pour tout $k \in K$, nous avons $\mathrm{dg}\left(\mathrm{T} \circ \mathrm{n}(\mathrm{k})-\mathrm{T} \circ \mathrm{n}^{\mathrm{b}}(\mathrm{k})\right) \leq \mathrm{dgT}-1$ VT $\mathrm{C}[\mathrm{N}]$, d'où pour toute mesure de probabilité $\mu$ sur $K$,

$$
\operatorname{dg}\left(Q_{\mu}^{b}-Q_{\mu}\right) T \leq \operatorname{dg} T-1 \quad ;
$$

et par suite $\operatorname{dg}\left(\left[I-Q_{\lambda_{2}}\left(I-Q_{m}\right)\right]^{-1}-\left[I-Q_{\lambda_{2}}^{b}\left(I-Q_{m}^{b}\right)\right]^{-1}\right) T \leq d g T-1$.

On en déduit alors que, $\operatorname{dg}\left(\mathrm{L}_{\lambda}^{\mathrm{b}}-\mathrm{L}_{\lambda}\right) \mathrm{T} \leq \mathrm{dgT}-3 \quad, \quad \forall \mathrm{FT} \in \mathbb{C}_{\ell(\lambda)}[\mathrm{N}]$.

Le lemme s'en déduit immédiatement. 
(2.16) Remarque.

Désignons par $\lambda^{n}$ la $\mathrm{n}^{\text {ième }}$ convolée de $\lambda$ dans $\mathrm{G}^{\mathrm{b}}$. Du

lemme (2.15), du corollaire $(2.8)$ et du théorème (2.12), il résulte

alors que les suites de mesures de probabilités,

$U_{n}^{b} \circ \pi_{1}\left(\lambda^{n}\right)=\pi_{1} \circ U_{n}^{b}\left(\lambda^{n}\right), U_{n}^{b} \circ \pi_{1}\left(\lambda^{n}\right)=\pi_{1}\left(\left[U_{n}^{b}(\lambda)\right]^{n \cdot}\right)$,

$\mathrm{u}_{\mathrm{n}}^{\mathrm{b}}\left(\overline{\lambda(a)}^{\mathrm{n}}\right)$ et $\mathrm{U}_{\mathrm{n}}^{\mathrm{b}}\left({\overline{\lambda(\mathrm{a})^{n}}}^{\mathrm{r}}\right)=\left[\mathrm{U}_{\mathrm{n}}^{\mathrm{b}}(\overline{\lambda(\mathrm{a})})\right]^{\mathrm{n}} \cdot$, convergent vaguement vers

la même mesure de probabilité, pour tout élément a de N vérifiant

la condition $(*)$ du corollaire $(2.8)$.

En particulier, 1orsque $\lambda=\lambda_{1} \otimes \lambda_{2}$ ou $\int_{N} A(u) \lambda_{1}(d u)=0$, pour toute forme 1 inéaire sur $N$ de degré 1 ,

$\mathrm{U}_{\mathrm{n}}^{\mathrm{b}} \circ \pi_{1}\left(\lambda^{\mathrm{n}}\right), \pi_{1}\left(\left[\mathrm{u}_{\mathrm{n}}^{\mathrm{b}}(\lambda)\right]^{\mathrm{n} \cdot}\right), \mathrm{U}_{\mathrm{n}}^{\mathrm{b}}\left(\bar{\lambda}^{\mathrm{n}}\right)$ et $\left[\mathrm{U}_{\mathrm{n}}^{\mathrm{b}}(\bar{\lambda})\right]^{\mathrm{n} \cdot}$,

convergent vaguement vers la même mesure de probabilité. 


\section{Démonstration de la proposition (2.6)}

Soit $\psi$ une mesure de probabilité sur $G \cdot Q_{\mu}$ opòre sur $\mathbb{C}_{\ell(\mu)}[N]$; nous notons $\Delta(\mu)$ l'ensemble des valeurs propres de $Q_{\mu}$ et pour a $\varepsilon(\mu)$, nous posons

$$
\begin{aligned}
& F_{\alpha}(\mu)=\left\{T \in \mathbb{C}_{\ell(\mu)}[N]: \exists k \in \mathbb{N},\left(Q_{\mu}-\alpha I\right)^{k} T=0\right\}, \\
& E_{\alpha}(\mu)=\left\{T \in \mathbb{C}_{\ell(\mu)}[N]: Q_{\mu} T=\alpha T\right\} ;
\end{aligned}
$$

nous avons

$$
\mathbf{C}_{\ell(\mu)}[\Lambda]=\underset{\alpha \in \Delta(\mu)}{\oplus} F_{\alpha}(\mu)
$$

(3.1) Lemme :

Soit $\mu$ une mesure de probabilité sur G, portée par $K$.

Alors
a) Tout élément de $\Delta(\mu)$ est de module $\leq 1$
b) Sì a $\dot{I}(\mu)$ avec $|\alpha|=1$, nous avons

$\mathrm{T} \in \mathrm{F}_{\alpha}(\mu) \Longleftrightarrow \mathrm{T} \in \mathrm{E}_{\alpha}(\mu) \Longleftrightarrow \mathrm{Q}_{\mathrm{k}} \mathrm{T}=\alpha \mathrm{T}, \quad \forall \mathrm{k} \in \mathrm{S}(\mu)$.

c) $\underset{\alpha \in \Delta(\mu)-\{1\}}{\oplus} F_{\alpha}(\mu)=E_{0}(m), F_{1}(\mu)=E_{1}(m)$

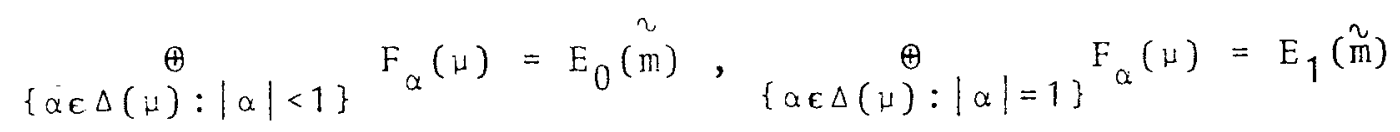

où $S(\mu)$ désigne le support de $\mu$, m(resp $\tilde{m})$ la mesure de Haar normalisée du sous-groupe fermé $K(\mu)$ (resp. $\hat{K}(\mu)$ ) engendré par $S(\mu)$ (resp. par $\left.S(\mu)[S(\mu)]^{-1}\right)$. 
Preuve du lemme $(3.1)$ :

Soit (, ) un produit scalaire quelconque sur l'espace vectoriel $\mathbb{C}[\mathrm{N}]$; en posant

$$
\left\langle\mathrm{T}, \mathrm{T}^{\prime}\right\rangle=\int_{\mathrm{K}}\left(\mathrm{Q}_{\mathrm{k}} \mathrm{T}, \mathrm{Q}_{\mathrm{k}^{\prime}} \mathrm{T}^{\prime}\right) \mathrm{dk} \quad\left(\mathrm{T}, \mathrm{T}^{\prime} \in \mathbb{C}[\mathrm{N}]\right) \text {, nous obtenons un }
$$

produit scalaire sur $\mathbb{C}[N]$, pour lequel les opérateurs $Q_{k}, k \in K$, sont unitaires (i.e. $\left\|\mathrm{Q}_{\mathrm{k}} \mathrm{T}\right\|^{2}=\left\langle\mathrm{Q}_{\mathrm{k}} \mathrm{T}, \mathrm{Q}_{\mathrm{k}} \mathrm{T}\right\rangle=\langle\mathrm{T}, \mathrm{T}\rangle=\|\mathrm{T}\|^{2}$,

$$
\text { YT } E \in \mathbb{C}[N]
$$

$$
\begin{aligned}
& \text { Soit } T \text { un élément de } \mathbb{C}[\mathrm{N}] \text {, nous arons } \\
& Q_{\mu} T=\int_{K} Q_{k} T \mu(d k) ;
\end{aligned}
$$

comme dans I'espace de Hilbert de dimension finie $\left(\mathbb{C}_{\mathrm{dgT}}[\mathrm{X}],<,>\right\}$ toute sphère de centre 0 est strictement convexe, il s'ensuit que

$$
|| Q_{v} T||<|| T||
$$

à moins que $Q_{k} T=T_{0}, \quad \forall k \in S(\mu), \operatorname{avec}|| T_{0}||=|| T||$.

$$
\begin{aligned}
& \text { L'affirmation a) du lemme s'en déduit immédiatement. } \\
& \text { Soit } T \in F_{\alpha}(\mu) \text {. Des relations } \\
& \underset{\tilde{m}}{Q_{\mu}} Q_{\mu}=Q_{\mu} Q_{\tilde{m}}=\underset{\tilde{m}}{Q_{k}} Q_{k}=Q_{k} Q_{m},
\end{aligned}
$$

vérifiées pour tout élément $k$ de $S(\mu)$, il résulte que les fonctions polynômes $\underset{\mathfrak{m}}{Q_{n}} \mathrm{~T}$ et. $\left(\mathrm{I}-\underset{\mathrm{m}}{Q_{n}} \mathrm{~T}\right.$ appartiennent respectivement à $F_{\alpha}\left(\varepsilon_{k}\right)=E_{\alpha}\left(\varepsilon_{k}\right)$ et $F_{\alpha}(\mu)$. Si $|\alpha|<1$, nous avons $E_{\alpha}\left(\varepsilon_{k}\right)=0$ car $Q_{k}$ est unitaire; par suite $\underset{\mathfrak{m}}{Q_{n} T}=0$. Supposons que $|\alpha|=1$ et désignons par $\ell$ le plus petit entier tel que $\left(Q_{\mu}-\alpha I\right)^{\ell}\left(I-Q_{\tilde{m}}\right) T=0$. Si $\ell \geq 1$, le polynôme $T^{\prime}=\left(Q_{\mu}-\alpha I\right)^{\ell-1}\left(I-Q_{\tilde{m}}\right) T$ appartient à $\mathrm{E}_{\alpha}(\mu)$, 
d'après ce qui précède nous avons alors $Q_{k^{\prime}} T^{\prime}=\alpha T^{\prime} \quad \forall k \cdot S(\mu)$; d'où $\left(I-\underset{m}{Q_{n}}\right) T^{\prime}=T^{\prime}=0$. 11 s'ensuit donc que $\ell=0 ; c^{\prime}$ est-à-dire $Q_{k} T=T \quad r_{k} \cdot \hat{k}(\mu)$. Comme nous savons en outre que $\underset{\mathfrak{m}}{Q_{n} T}$ (qui est égal à I) appartient à $E_{\alpha x}\left(\varepsilon_{k}\right)$, I'affirmation b) ainsi que les deux dernières égalités de c) sont prouvées.

Enfin si $T: F_{\alpha}(\mu)$, nous avons $\left(Q_{\mu}-\alpha I\right)^{2} T=0$ : pour un certain entier $\ell$. D'où $(1-\alpha)^{\ell} Q_{m} T=0$ et $Q_{m} T=0$ si $\alpha \neq 1$. Si $T=F_{1}(\mu)$, d'après b) nous avons $Q_{k} T=T, V k: S(\mu)$, et par suite $Q_{m} T=T$. Le lemme est ainsi démontré.

Pour simplifier l'écriture, nous notons Q (resp. P) l'opérateur $Q_{\lambda}$ (resp. $\left.Q_{\lambda_{2}}\right)$. Si $r<\mathbb{N}^{*}$ et $\alpha=\mathbb{N}^{r}$, nous notons $\alpha_{1}, \ldots$, $\alpha_{r}$ les composantes de $\alpha$ et nous posons ||$\alpha||=\alpha_{1}+\ldots+\alpha_{r}$. D'autre part, si $t<\mathbb{R}_{+}$, nous désignons par $\lfloor t]$ la partie entière de $t$.

$$
\text { D'après 1e lemme }(2.4) \text {, nous avons } \mathrm{dg}(\mathrm{Q}-\mathrm{P}) \mathrm{T} \leq \mathrm{dg} \mathrm{T}-1
$$
$\forall T: C_{\ell(\lambda)}[N]$; par suite en écrivant $Q=(Q-P)+P$, nous avons

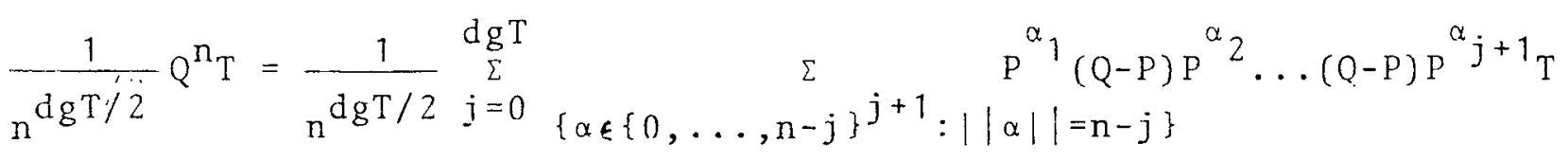

\section{Posons}

$$
\begin{aligned}
& \mathrm{R}_{0}^{0}=\mathrm{I}-\mathrm{Q}_{\mathrm{m}}, \mathrm{R}_{0}=\left(\mathrm{I}-\mathrm{Q}_{\mathrm{m}}\right) \mathrm{P}\left(\mathrm{I}-\mathrm{Q}_{\mathrm{m}}\right), \\
& \mathrm{R}_{1}^{0}=\mathrm{R}_{1}=\mathrm{Q}_{\mathrm{m}} \text { et } \mathrm{P}^{0}=\mathrm{Q}^{0}=\mathrm{I} ;
\end{aligned}
$$

pour tout entier naturel p, nous avons

$$
\begin{aligned}
& Q^{p}=R_{0}^{p}+R_{1}^{p}, R_{0}^{p}=\left(I-Q_{m}\right){ }^{p}\left(I-Q_{m}\right) \text { et } R_{1}^{p}=Q_{m} \text {. } \\
& \frac{1}{n^{\mathrm{dgT} / 2}} \mathrm{Q}^{\mathrm{n}_{\mathrm{T}}} \text { s'écrit alors } \sum_{j=0}^{\mathrm{dgT}} i<\{0,1\}^{j+1} \gamma_{n}(j, i) \text {, } \\
& \text { où } \gamma_{n}(j, i)=\frac{1}{n^{d g T / 2}}\left\{\alpha \dot{\varepsilon}\{0, \ldots, n-j\} j^{j+1}:|| \alpha||=n-j\right\} \text {, } \\
& \text { avec } \phi(j, i, \alpha)=R_{i_{1}}^{\alpha}(Q-P) R_{i}^{\alpha}{ }_{2}^{2} \ldots(Q-P) R_{i}^{\alpha}{ }_{j+1}+1 T \text {. }
\end{aligned}
$$


3.2 Lemme.

Soient $j \in\{0, \ldots, d g T\}$ et $i<\{0,1\}^{j+1}$ avec

||$i|| \leq[(\mathrm{dg} T+1) / 2]$. Alors 1 a suite de fonctions polynomes $\gamma_{n}(j, i)$ converge vers zéro, uniformément sur tout compact.

Preuve. Notons $\sigma(1), \ldots, \sigma(s),(\operatorname{resp} \cdot \sigma(s+1), \ldots, \sigma(j+1))$, avec $s \leq[(\operatorname{dg} T+1) / 2]$, les entiers $\ell$ de $\{1, \ldots, j+1\}$ tels que $i_{\ell}=1$ (resp. $\left.i_{\ell}=0\right)$. Nous avons

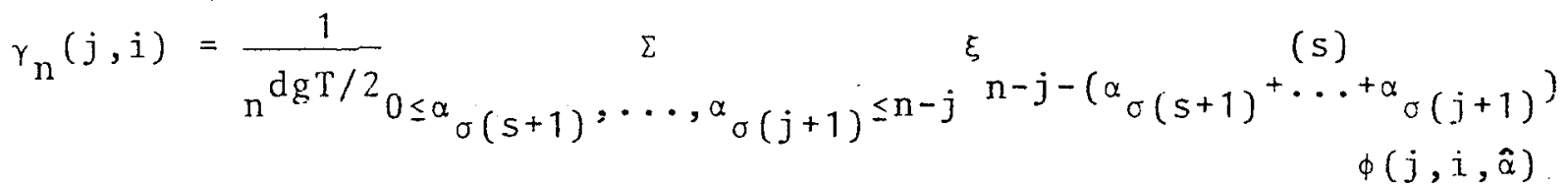

où, pour tout $\ell \in \mathbb{N}$ et tout $s \in \mathbb{N}^{*}, \xi_{\ell}(s)=\operatorname{card}\left\{B \in \mathbb{N}^{s}:|| \beta||=\ell\right\}$ et $\hat{\alpha}$ est l'élément de $\mathbb{N}^{j+1}$ qui se déduit de $\alpha$ en remplaçant les composantes $\alpha_{\sigma(i)}, i \in\{1, \ldots, s\}$, par zéro.

D'après le lemme (3.1), les valeurs propres de l'opérateur $R_{0}$ sont de module $\leq 1$ et différentes de 1 ; il s'ensuit que $1 \mathrm{a}$ suite d'opérateurs de $\mathbb{C}[N],\left\{_{p} \sum_{0}^{n} R_{0}^{p}\right\}_{n \geq 1}$, est bornée. D'autre part pour tout $\ell \in \mathbb{N}$ et tout $s: \mathbb{N}^{*}, \xi_{\ell}(s)$ est un polynome de degré $(s-1)$ en $\ell$ dont le terme de plus haut degré est $\ell^{s-1} /(s-1) !$. Le lemme (3.2) est alors clair.

$3.3 \quad$ Lemme

Soient $j:\{0, \ldots, d g T\}$ et $i=\{0,1\}\}^{j+1}$ avec

||$i \mid p[(\operatorname{dgT}+1) / 2]+1$. Alors $\gamma_{n}(j, i)$ est une constante qui est non nulle seulement si dgT est pair, $\| \mathrm{i}||=\mathrm{dgT} / 2+1$, $i_{1}=i_{j+1}=1$ et $i$ ne possède pas deux composantes nulles consécu= tives.

Preuve. Désignons par $N(i)$ le nombre de couples $\left(i_{k}, i_{k+1}\right), 0 \leq k \leq j$, égaux à $(1,1)$; nous avons

$$
N(i)=\sum_{1 \leq k \leq j}\left[\left(i_{k}+i_{k+1}\right) / 2\right] \geq 2|| i||-j-2 .
$$

Comme 1 'opérateur $R_{1}(Q-P) R_{1}=Q_{m}\left(Q_{\bar{\lambda}}^{-} I\right) Q_{m}$ baisse le degré de tout polynome de $\mathbb{C}_{\ell(\lambda)}\left[\mathrm{N}^{\mathrm{N}}\right]$ de deux unitës, nous avons, pour tout 
$\alpha:\{0, \ldots, n-j\}\}^{j+1}$

$$
\begin{aligned}
\operatorname{dg} \phi(j, i, \alpha) & \leq \operatorname{dg} T-(j+N(i)) \\
& \leq \operatorname{dg} T-2|| i||+2
\end{aligned}
$$

qui montre que $\phi(j, i, \alpha)$ est une constante qui est non nulje seulement si dgT est pair. $\|\mathrm{i}\|=\operatorname{dgT} / 2+1$ et $N(\mathrm{i})=2|| i \|-j-2$ Le lemme (3.3) s'en déduit immédiatement.

Des lemmes (3.2) et (3.3), il résulte que si dgT est impair la suite de fonctions polynomes $\left\{\frac{1}{n^{\mathrm{dgT} / 2}} Q^{\mathrm{n}} \mathrm{T}\right\}{ }_{\mathrm{n} \geq 1}$ converge, uniformément sur tout compact, vers zéro. ${ }^{\mathrm{dg} T / 2} \mathrm{n} \geq 1$

Supposons que dgT soit pair. Pour tout entier $k$, notons $S^{k}$ (resp. $\left.V, V^{0}\right)$ I'opérateur $Q_{m}(Q-I) R_{0}^{k}(Q-I) Q_{m}$ (resp. $Q_{m}(Q-I) Q_{m}$, I) . Des lemmes (3.2) et (3.3), il résulte que $\frac{1}{\mathrm{dg} T / 2} Q^{\mathrm{n}_{\mathrm{T}}}$ s'écrit $\delta_{1}(\mathrm{n})+\delta_{2}(\mathrm{n})$, où $\delta_{1}(\mathrm{n})$ est une suite de fonctions polynomes convergeant, uniformément sur tout compact, vers zéro et

avec

$$
\begin{aligned}
& \delta_{2}(n)=\sum_{s=1}^{\operatorname{dgT} / 2} \underset{\left\{\beta \in \mathbb{N}^{s+1}:|| \beta||=\operatorname{dgT} / 2-s\right\}}{\psi_{n}(s, \beta)}
\end{aligned}
$$

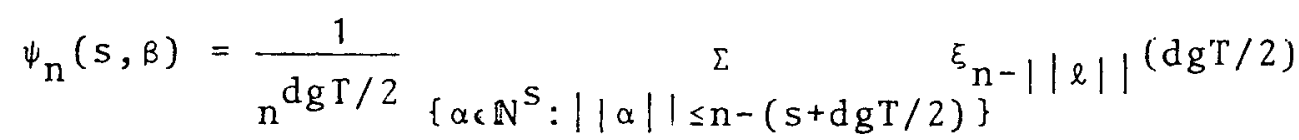

$$
\begin{aligned}
& V^{\beta}{ }_{1} S^{\alpha}{ }_{1} V^{\beta}{ }_{2} \ldots V^{\beta} S_{S}{ }^{\alpha} S_{V}{ }^{\beta} S+1_{T} \text {. }
\end{aligned}
$$

Désignons par $a_{1}, \ldots, a_{q}$ les différentes valeurs propres de $P$ de module 1 et distinctes de 1 . Nous avons (lemme (3.1))

$$
R_{0}=\left(I-Q_{m}\right) P\left(I-Q_{m}\right)=\left(I-Q_{n}\right) P\left(I-\underset{m}{Q_{n}}\right)+\underset{i=1}{q} a_{i} \pi_{i}
$$

où $\pi_{i}$ désigne la projection de $C[N]$ sur $F_{a_{i}}\left(\lambda_{2}\right)=E_{a_{i}}\left(\lambda_{2}\right)$; et par suite, pour tout élément $k$ de $N^{*}$,

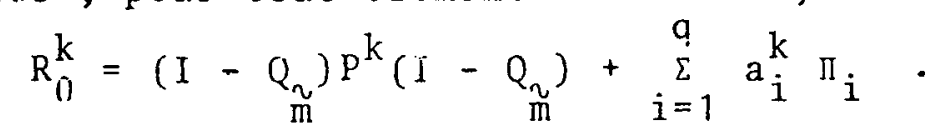

Pour tout entier naturel $k$, nous posons

$$
S_{0}^{k}=Q_{m}(Q-I)\left(I-Q_{\tilde{m}}\right) P^{k}\left(I-Q_{\tilde{m}}\right)(Q-I) Q_{m}
$$




$$
S_{i}^{k}=a_{i}^{k} Q_{m}(Q-I) \pi_{i}(Q-I) Q_{m}, 1 \leq i \leq q .
$$

Soient $s \in\{0, \ldots, \mathrm{dgT} / 2\}$ et $B: \mathbb{N}^{s+1}$ avec

||$\beta||=\mathrm{dgT} / 2-\mathrm{s}$. Nous avons

$$
\psi_{\mathrm{n}}(s, \beta)=\underset{j \in\{0, \ldots, q\} s^{\tau_{n}}(s, \beta, j)}{\sum}
$$

avec

$$
\begin{aligned}
& \zeta_{n}(s, \beta, j)=\frac{1}{n^{\operatorname{dgT} / 2}}\left\{\alpha \leq \mathbb{N}^{s}:|| \alpha|| \leq n-(s+\operatorname{dgT} / 2)\right\}^{\xi} \xi_{n}-\left.|\alpha|\right|^{(\operatorname{dgT} / 2)} \\
& v^{\beta} 1 S_{j_{1}}^{\alpha} \ldots v^{\beta} s S_{j_{s}}^{\alpha}{ }_{s}{ }^{\beta}{ }^{\beta}+1_{T} \\
& =\frac{1}{\mathrm{dgT} / 2} \sum_{k=0}^{n-(s+\operatorname{dgT} / 2)} \zeta_{n-k}(\operatorname{dgT} / 2) n_{k}(s, \beta, j) \\
& \text { où } \quad n_{k}(s, \beta, j)=\sum_{\left\{\alpha: \mathbb{N}^{s}:|| \alpha||=k\right\}} v^{\beta}{ }_{1} S_{j}^{\alpha}{ }_{1} \ldots V^{\beta} s^{\alpha} s_{j}^{\alpha} s_{V}^{\beta} s+1 T .
\end{aligned}
$$

En notant que

$\left(I-P\left(I-Q_{m}\right)\right)^{-1}=Q_{m}+\sum_{1 \geq 0}\left(I-\underset{m}{Q_{n}}\right) P^{1}\left(I-Q_{m}\right)+\sum_{i=1}^{q} \Pi_{i} /\left(1-a_{i}\right)$,

la proposition (2.6) résulte alors du lemme suivant :

\section{$3.4 \quad$ Lemme}

Soient $p$ et $q$ deux entiers $\geq 1 ; x_{1}, \ldots, x_{q}$ des nombres complexes différents de 1 et de modules $1 ; u$ une fonction réelle de ${ }_{N}^{p}$ telle que $\sum_{1<\mathbb{N}^{p}} \mathrm{u}(1)<+\infty$. Alors, pour tout entier $\mathrm{r} \geq 1$,

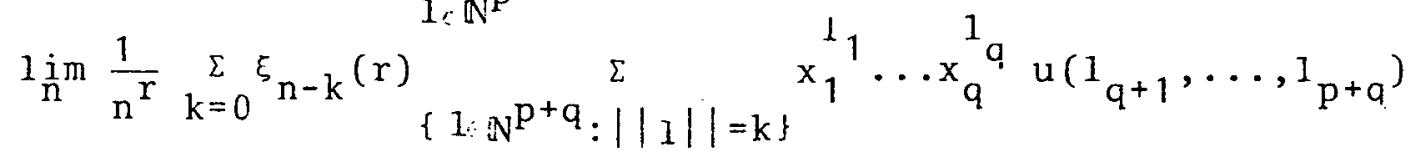

$$
\begin{aligned}
& =\frac{1}{1-x_{1}} \cdots \frac{1}{1-x_{q}} \frac{1}{r !} \sum_{1 \cdot \mathrm{N}^{p}} \mathrm{u}(1) \\
& \lim _{n} \frac{1}{n^{r}} \sum_{k=0} \xi_{n-k}(r) \sum_{\left\{1: \mathbb{N}^{p}:|| 1||=k\right\}}^{\sum} x_{1}^{1} \ldots x_{q}^{1} q=\frac{1}{r !} \frac{1}{1-x_{1}} \cdots \frac{1}{1-x_{q}} \\
& \lim \frac{1}{n^{r}} \sum_{k=0} \xi_{n-k}(r) \quad \sum_{\left\{1 . N^{p}:|| 1||=k\right\}} u\left(1_{1}, \ldots, 1_{p}\right)=\frac{1}{r !} \sum_{1 \in \mathbb{N}} p(1) .
\end{aligned}
$$

Preuve. Posons

$$
B(k)=\sum_{\left\{1: N^{p+q}:\|1\|=k\right\}} x_{1}^{1} \ldots x_{q}^{1} q u\left(1_{q+1}, \ldots, 1_{p+q}\right) ;
$$


nous avons $B(k)=\sum_{s=0}^{k} \sigma(s) \tau(k-s)$, en posant, pour tout entier S,

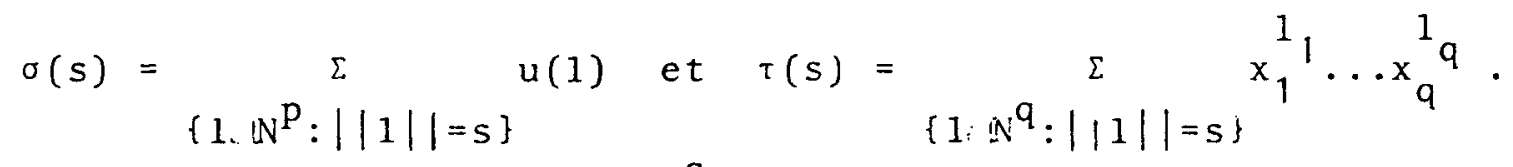

Considérons le polynome $Q(x)=\prod_{i=1}^{q}\left(1-x_{i}\right)$; il est facile de voir

que

$$
\begin{aligned}
& \tau(s)=\sum_{i=1}^{q} x_{i}^{s+1} / Q^{\prime}\left(1 / x_{i}\right) \quad \text { et par suite } \\
& \beta(k)=\sum_{i=1}^{q}\left(x_{i} / Q^{\prime}\left(1 / x_{i}\right)\right) \sum_{s=0}^{k} \sigma(s) x_{i}^{k-s} .
\end{aligned}
$$

$D^{\prime}$ où

$$
\begin{aligned}
\sum_{k>0} B(k) & =\sum_{i=1}^{q}\left(x_{i} /\left(1-x_{i}\right) Q^{\prime}\left(1 / x_{i}\right)\right) \sum_{s \geq 0} \sigma(s) \\
& =(1 / Q(1)) \sum_{s \geq 0} \sigma(s) .
\end{aligned}
$$

Le lemme (3.4) résulte alors du lemme élémentaire suivant.

3. 5 Lemme

Soient $x$ un nombre complexe différent de 1 , de module 1 et $\left\{\beta_{k}\right\}_{k \geq 0}$ une série convergente. Alors, pour tout entier $r>0$,

$$
\begin{aligned}
\lim \frac{1}{n} \sum_{k=0}^{n}(n-k)^{r} x^{k} & =\frac{1}{r !} \frac{1}{1-x} \\
\lim \frac{1}{n^{r}} \sum_{k=0}^{n}(n-k)^{r} \beta_{k} & =\frac{1}{r !} \sum_{k \geq 0}^{\sum} \beta_{k} .
\end{aligned}
$$


4. Démonstration du théorème $(2.12)$

Nous posons $r=\sup \left\{d g x_{i}, 1 \leq i \leq p\right\} ; r$ est indépendant du choix du système de fonctions coordonnées $\left\{x_{i}\right\}_{1 \leq i \leq p}$ de 1 'e.v. $\quad N$ Pour faciliter la lecture, nous distinguons deux cas.

1er cas

$=== \pm==$

Nous supposons que $\lambda$ vérifie, outre l'hypothèse iii) de $(2.1)$, la condition $m(\lambda) \geq 2 \sup (r, 2)$. [On notera que d'après l'inégalité de Hölder̀, cette dernière hypothèse entraîne la condition (*) de $(2.11)]$.

La démonstration du théorème se fait en trois étapes.

lère étape. Elle est suggérée par la remarque (2.14).

Pour prouver le théorème (2.12), nous pouvons supposer que pour tout $t>0, \mathrm{U}_{t}^{\mathrm{b}}$ est un automorphisme de $G$ (i.e. $G=G^{b}$, voir (2.14)). En effet, nous avons:

\section{(4.1) Proposition:}

Soit $\left(X_{i}\right)_{i>1}$ une suite de v.a. indépendantes, à valeurs dans $N \times K$, et de loi commune $\lambda$. Alors la suite de v.a.

$$
\Delta_{n}=u_{n}^{b} \circ \pi_{1}\left(x_{1} \ldots x_{n}\right)-u_{n}^{b} \circ \pi_{1}\left(x_{1} \cdot \ldots \cdot x_{n}\right)
$$

converge vers zéro dans $\mathrm{L}^{2}$.

Preuve de la proposition (4.1):

Désignons par $M$ le produit direct des groupes de lie nilpotent simplement connexe $N$ et $N^{b}$; par $n \otimes n^{b} 1^{\text {"hhomomorphisme }}$ de $K$ dans le groupe des automorphismes de $M$ défini par 
$\left[n \otimes n^{b}(k)\right](u, v)=\left(n(k)(u), n^{b}(k)(v)\right),(u, v)<\times \times N^{b}$;

et par $H=M x_{n{ }_{n}} b K$ le produit semi-direct de $M$ par $K$ associé à $n \otimes n^{b}$.

Si $\mu$ est une mesure de probabilité sur $N \times N \times K$, nous notons $\sim_{\mu}$ l'opérateur de transition sur $M$, identifié à $H / K$, associé à $\mu$ (voir (1.3)). Soit $X=(Y, U)$ une v.a., à valeurs dans dans $\lambda \times K$, de $10 \mathrm{i} \lambda$; nous notons $\lambda^{\prime}$ la loi de la $v . a .(Y, Y, U)$, à valeurs dans $N \times N \times K$.

Avec ces notations, nous avons, pour tout i $\varepsilon\{1, \ldots, p\}$, $\mathbb{E}\left[x_{i}^{2}\left(\Delta_{n}\right)\right]=\frac{1}{n} \frac{1}{d g} x_{i} Q_{\lambda}^{n}, T_{i}(0)$,

où $T_{i}$ désigne la fonction polynome sur $N \times N$ définie par $T_{i}(u, v)=\left(x_{i}(u)-x_{i}(v)\right)^{2}, u$ et $v \in N$.

Nous définissons une notion de degré sur $\mathbb{C}[M]=\mathbb{C}\left[\begin{array}{lll}N & \times\end{array}\right]$, en attribuant un degré à toute fonction polynome $T$ de $\mathbb{C}\left[\begin{array}{lll}N & \mathrm{~N}\end{array}\right]$ de la forme $\mathrm{T}_{1} \otimes \mathrm{T}_{2}$, où $\mathrm{T}_{1}$ et $\mathrm{T}_{2}$ sont des éléments de $\mathbb{C}[\mathrm{N}]$, (i.e. $\left.\mathrm{T}_{1} \otimes \mathrm{T}_{2}(\mathrm{u}, v)=\mathrm{T}_{1}(u) \mathrm{T}_{2}(v), \mathrm{F}(\mathrm{u}, v) \in \mathrm{N} \times \mathrm{N}\right) ;$ le degré de $\mathrm{T}$, noté dgT, est par définition égal à $\mathrm{dgT}_{1}+\mathrm{dgl}_{2} \cdot$ Le couple $\left(\mathrm{H}, \lambda^{\prime}\right)$ vérifie alors les hypothèses de (2.1).

Considérons $1 a$ base $b \otimes b=\left\{\left(e_{i}, 0\right),\left(0, e_{j}\right), 1 \leq i, j \leq p\right\}$ de $N \times N$. Avec les notations de 1 a remarque $(2.14)$, $N^{b \otimes b}$ est 1 e produit direct de $\mathrm{N}^{\mathrm{b}}$ par lui-même et $\mathrm{H}^{\mathrm{b} \otimes b}$ est le produit semi-direct de $M^{b}$ par $K$ associé à $n^{b} \otimes n^{b}$.

Le lemme (2.15), appliqué au couple $\left(H, \lambda^{\prime}\right)$, nous dit que $\lim _{n} \frac{1}{\mathrm{dgx}} \chi_{\lambda}^{\mathrm{n}}, \mathrm{T}_{i}=\lim _{\mathrm{n}} \frac{1}{\mathrm{dgx_{i }}}\left(\ell_{\lambda}^{\mathrm{b} \otimes \mathrm{b}}\right)^{\mathrm{n}} \mathrm{T}_{i}, \forall i \in\{1, \ldots, \mathrm{p}\}$, où $Q_{\lambda}^{\mathrm{b} Q}$ désigne $I^{\prime}$ 'opérateur de transition sur $M^{\mathrm{b} Q \mathrm{~b}}$, identifié à 
$H^{b \otimes b} / K$, associé à $\lambda^{\prime}$. Or nous avons, pour u $N^{\prime}$ et $i \epsilon\{1, \ldots, p\}$ $\left({\underset{\sim}{(O)}}_{\lambda}^{b}{ }^{b}\right)^{n} T_{i}(u)=E\left[\left[x_{i} \circ \pi_{1}\left(x_{1} \cdot \ldots \cdot x_{n} \cdot(u, e)\right)-x_{j} \quad \circ \pi_{1}\left(x_{1} \cdot \ldots \cdot x_{n} \cdot(u, e)\right)\right]^{2}\right]=0$ II s'ensuit que $\lim _{n} \frac{1}{\mathrm{dgx}_{i}} \ell_{\lambda}^{n}, \mathrm{~T}_{i}=0$ et la proposition (4.1) est prouvée.

2è étape. Nous supposons donc que $V t>0, U_{t}^{b}$ est un automorphisme de G.

Soit $\left(X_{i}\right)_{i>1}$ une suite de va. indépendantes, à valeurs dans $N \times K$, de 10 i commune $\lambda$. Nous posons, pour tout entier naturel $\mathrm{n} \geq 1$

$$
\begin{aligned}
& S_{n}(0)=0 \\
& S_{n}\left(\frac{k}{n}\right)=\pi_{1} \circ U_{n}\left(x_{1} \ldots x_{k}\right)=\pi_{1}\left(U_{n}\left(X_{1}\right) \ldots U_{n}\left(X_{n}\right)\right), \forall k:\{1, \ldots, n\} ;
\end{aligned}
$$

puis

$$
S_{n}(t)=(1-\{n t\}) S_{n}\left(\frac{[n t]}{n}\right)+\{n t\} \quad S_{n}\left(\frac{[n t]+1}{n}\right) \text {, Ht } \in[0,1] \text {, }
$$

où $[n t]$ désigne la partie entière de nt et $\{n t\}=n t-[n t]$. Pour tout $u \in N$, nous posons $\left.|| u\right|^{2}=\sum_{i=1}^{p} x_{i}^{2}(u)$.

(4.2) Proposition.

$$
\begin{aligned}
& \text { Pour tous réels } s \text { et } t \text { de }[0,1], \\
& E\left[|| S_{n}(t)-\left.S_{n}(s)\right|^{2}\right] \leq C|t-s|,
\end{aligned}
$$

où $C$ est une constante $>0$ indépendante de $n, t$ et $s$.

Preuve de la proposition (4.2) -

Nous commençons par prouver qu'il suffit de montrer la propo- 
sition pour des réels $s$ et $t$ de la forme $\frac{k}{n}$ avec $k \in\{0, \ldots, n\}$.

Admettons donc un instant que la proposition soit vraie pour les réels de cette forme et considérons deux réels $s$ et $t$ de $[0,1]$ vérifiant $s<t$.

Nous avons

$$
s_{n}(t)-s_{n}(s)=\{n t\} u+v+(1-\{n s\}) w,
$$

avec

$$
\begin{aligned}
& u=s_{n}\left(\frac{[n t]+1}{n}\right)-s_{n}\left(\frac{[n t]}{n}\right), \\
& v=s_{n}\left(\frac{[n t]}{n}\right)-s_{n}\left(\frac{[n s]+1}{n}\right),
\end{aligned}
$$

et

$$
w=S_{n}\left(\frac{[n s]+1}{n}\right)-s_{n}\left(\frac{[n s]}{n}\right)
$$

De la relation

$$
\begin{aligned}
& \|a x+b y+c z\|^{2} \leq(a+b+c)\left(a|| x\left\|^{2}+b\right\| y\left\|^{2}+c|| z\right\|^{2}\right), a, b, c>0, x, y, z \in N, \\
& \text { il résulte que l'on a } \\
& E\left[|| s_{n}(t)-\left.S_{n}(s)\right|^{2}\right] \leq \frac{3 C}{n}(\{n t\}+|[n t]-[n s]-1|+1-\{n s\}) \text {. } \\
& \text { D' où } \\
& E\left[\left\|S_{n}(t)-S_{n}(s)\right\|^{2}\right] \leq 3 C(t-s) \quad, \quad s i[n t]-[n s] \geq 1 .
\end{aligned}
$$$$
\text { Si }[n t]=[n s] \text {, nous avons }
$$$$
s_{n}(t)-s_{n}(s)=n(t-s)\left(s_{n}\left(\frac{[n t]+1}{n}\right)-s_{n}\left(\frac{[n t]}{n}\right)\right)
$$$$
\text { et }
$$$$
E\left[\left\|s_{n}(t)-s_{n}(s)\right\|^{2}\right] \leq C n(t-s)^{2}
$$$$
\leq C(t-s)
$$ 
car $[n t]=[n s]$ implique que $n(t-s) \leq 1$.

Ceci dit soient $k$ et $\ell$ deux entiers de $\{0, \ldots, n\}$ tels que $\ell<k$. Pour tout i $\varepsilon\{1, \ldots, p\}$, posons

$$
T_{i}(u, v)=x_{i}(u v)-x_{i}(u),(u, v \in N)
$$

nous avons

$$
T_{i}(u, v)=x_{i}(v)+P_{i}(u, v),
$$

avec $\operatorname{dg} \mathrm{P}_{i} \leq \mathrm{dg} \mathrm{x}_{i}$, val $\mathrm{P}_{i} / \mathrm{u}$ et val $\mathrm{P}_{i} / \mathrm{v} \geq 1$.

Par suite

$E\left[\left[x_{i}\left(S_{n}\left(\frac{k}{n}\right)-S_{n}\left(\frac{l}{n}\right)\right)\right]^{2}\right]=\mathbb{E}\left[\left[x_{i}\left(\left[S_{n}\left(\frac{l}{n}\right)\right]^{-1} S_{n}\left(\frac{k}{n}\right)\right)+\right.\right.$

$$
\left.+P_{i}\left(S_{n}\left(\frac{\ell}{n}\right),\left[S_{n}\left(\frac{\ell}{n}\right)\right]^{-1} S_{n}\left(\frac{k}{n}\right)\right)\right]^{2} !
$$

or $\left[S_{n}\left(\frac{\ell}{n}\right)\right]^{-1} S_{n}\left(\frac{k}{n}\right)$ s'écrit $n \circ \pi_{2}\left(x_{1} \ldots x_{\ell}\right)\left(\sum_{\ell, k}(n)\right)$,

où $\sum_{\ell, k}(n)$ est une v.a. indépendante de $S_{n}\left(\frac{l}{n}\right)$ et ayant la même loi que $S_{n}\left(\frac{k-\ell}{n}\right)$. La proposition (4.2) résulte alors du lemme suivant.

(4.3) Lemme.

Pour tout élément $\mathrm{T}$ de $\mathbb{C}_{\ell(\lambda)}[\mathrm{N}]$ de la forme $\mathrm{x}_{1}^{\alpha_{1}} \ldots x_{\mathrm{p}}^{\alpha} \mathrm{p}$, où $\alpha_{1}, \ldots, \alpha_{p} \in \mathbb{N}$, et pour tout élément $k$ de $\{0, \ldots, n\}$, nous avons

$$
\left|E\left[T\left(S_{n}\left(\frac{k}{n}\right)\right)\right]\right| \leq C(T)\left(\frac{k}{n}\right) \operatorname{dgT} / 2
$$

où $\mathrm{C}(\mathrm{T})$ est une constante $>0$ indépendante de $\mathrm{k}$ et $\mathrm{n}$.

Preuve du lemme (4.3). 


$$
\begin{aligned}
& E\left[T\left(S_{n}\left(\frac{k}{n}\right)\right)\right]=\frac{1}{n} \frac{1}{d g T / 2} Q_{\lambda}^{k} T(0) \\
& =\left(\frac{k}{n}\right)^{d g T / 2}\left(\frac{1}{k^{d g T / 2}} Q_{\lambda}^{k} T(0)\right) . \\
& \text { D'après la proposition (2.6), la suite } \frac{1}{k^{\mathrm{dgT} / 2}} Q_{\lambda}^{k} \mathrm{~T}
\end{aligned}
$$

est convergente. Le lemme est ainsi prouvé.

(4.4) De la proposition (4.2), il résulte que le processus $S_{n}(t)$ vérifie les hypothèses du corollaire 1 du chapitre I, sec. 6 de [ 8 ] Désignons par $\mathcal{B}([0,1])$ la tribu des boréliens de $[0,1]$ et par $\rho$ la mesure de Lebesgue de $[0,1]$. Nous savons alors que pour toute suite d'entiers, on peut extraire une sous-suite $\left\{n_{k}\right\}_{k \geq 1}$ pour laquelle il est possible de construire des processus $x_{n_{k}}\left(t, \omega^{\prime}\right)$ et $x_{\infty}\left(t, \omega^{\prime}\right)$ sur $l^{\prime}$ espace probabilisé $\left(\Omega^{\prime}=[0,1], \mathcal{B}([0,1]), \rho\right)$ tels que

i) pour tout entier $\ell$ et tous réels $t_{1}, \ldots, t_{\ell}$, $\left(x_{n_{k}}\left(t_{1}\right), \ldots, x_{n_{k}}\left(t_{\ell}\right)\right)$ e.t $\left(s_{n_{k}}\left(t_{1}\right), \ldots, s_{n_{k}}\left(t_{\ell}\right)\right)$ ont même 10i, pour tout entier $k$.

ii) pour tout $t \in[0,1], x_{n_{k}}(t)$ converge en probabilité vers $x_{\alpha}(t)$, quand $k$ tend vers l'infini.

3ème étape. Désignons par $\left\{n_{k}\right\} k 1$ une suite d'entiers pour laquelle il est possible de construire des processus $x_{n_{t}}(t)$ et $x_{\infty}(t)$ ayant les propriétés i) et $\left.i i\right)$ de (4.4). Alors le processus $x_{\infty}(t)$ vérifie la proposition suivante:

(4.5) Proposition.

Pour toute fonction sur $N$, de classe $C^{2}$, à support 
compact,

$\mathbf{E}_{\rho}\left[f\left(x_{\infty}(t)\right)\right]=f(0)+\int_{0}^{t} E_{\rho}\left[A f\left(x_{\infty}(s)\right)\right] d s$, Yt $c[0,1]$, où $\mathbb{E}_{\rho}$ désigne 1 'espérance associée à la mesure $\rho$.

Preuve de la proposition (4.5).

En posant $S_{k}^{\prime}(t)=S_{n_{k}}(t)$ et $x_{k}^{\prime}(t)=x_{n_{k}}(t)$, on se ramène au cas où $\left\{n_{k}\right\}_{k \geq 1}$ est 1 a suite des entiers naturels. Pour alléger 1'écriture, nous nous plaçons donc dans cette situation.

\section{Pour toute fonction $f$ sur $N$, de classe $C^{1}$, à support} compact, et pour tout élément $v$ de $N$, nous posons

$$
D_{v} f(u)=\lim _{t \rightarrow 0} \frac{f(u(t v))-f(u)}{t}, \quad\left(u \in N^{\prime}\right)
$$

Nous avons

$$
D_{v} f=\sum_{i=1}^{p} x_{i}(v) D_{i} f \quad, \quad \text { où } \quad D_{i}=D_{e_{i}}, \quad i \in\{1, \ldots, p\} ;
$$

et (formule de Taylor), pour tous $u, v \in N$, il existe $\theta \in] 0,1[$ tel

que

$$
f(u v)=f(u)+D_{v} f(u)+\frac{1}{2} D_{v}^{2} f(u(\theta v))
$$

Choisissons une partition $t_{0}=0<t_{1}<\ldots<t_{q}=t$ de $[0, t]$ et écrivons, pour tout entier naturel $\mathrm{n}$,

$$
\begin{aligned}
f\left(S_{n}(t)\right)-f(0)=f\left(S_{n}(t)\right)-f\left(S_{n}\left(\frac{[n t]}{n}\right)\right)+ & \sum_{j=1}^{q}\left\{f\left(S_{n}\left(\frac{[n t j]}{n}\right)\right)-\right. \\
& \left.-f\left(S_{n}\left(\frac{[n t j-1}{n}\right)\right)\right\} .
\end{aligned}
$$

D'après 1 a proposition (4.2), il est clair que $\lim _{n} \mid \mathbb{E}\left[f\left(S_{n}(t)\right)-f\left(S_{n}\left(\frac{[n t]}{n}\right)\right)\right]=0$.

$$
\text { Posons } \sum_{j}(n)=\left[s_{n}\left(\frac{\left[n t_{j}-1\right]}{n}\right)\right]^{-1} s_{n}\left(\frac{\left[n t_{j}\right]}{n}\right), j \in\{1, \ldots q\} \text {; }
$$

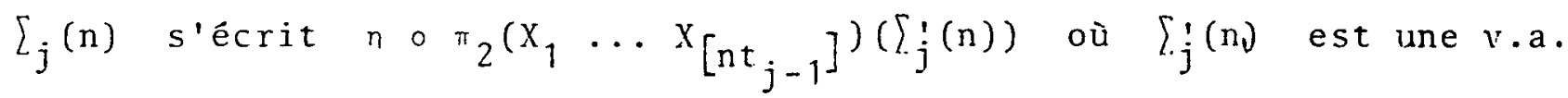


indépendante de $S_{n}\left(\frac{\left[\mathrm{nt}_{j-1}\right]}{n}\right)$ et de même loi que $S_{n}\left(\frac{\left[\mathrm{nt}_{j}\right]-\left[n t_{j-1}\right]}{n}\right)$

D'après la formule de Taylor, nous avons

$f\left(S_{n}\left(\frac{\left[n t_{j}\right]}{n}\right)\right)-f\left(S_{n}\left(\frac{\left[n t_{j-1}\right]}{n}\right)\right)=\alpha_{j}(n)+\frac{1}{2} \beta_{j}(n)$,

avec

$$
\begin{aligned}
& \alpha_{j(n)}=\sum_{i=1}^{p} x_{i}\left(\Sigma_{j}(n)\right) D_{i} f\left(S_{n}\left(\frac{[n t j-1]}{n}\right)\right)+\frac{1}{2} \sum_{1 \leq i, \ell \leq p} x_{i}\left(\Sigma_{j}(n), x_{\ell}\left(\Sigma_{j}(n)\right)\right. \\
& D_{i} D_{\ell} f\left(S_{n}\left(\frac{\left[n t_{j-1}\right]}{n}\right)\right)
\end{aligned}
$$

et

$$
\begin{aligned}
B_{j}(n)=\sum_{1 \leq i, \ell \leq p} x_{i}\left(\Sigma_{j}(n)\right) x_{\ell}\left(\Sigma_{j}(n)\right)\left[D_{i} D_{\ell}\right. & f\left[S_{n}\left(\frac{\left[n t_{j}-1\right]}{n}\right)\left(\theta \Sigma_{j}(n)\right)\right]- \\
& \left.-D_{i} D_{\ell} f\left[S_{n}\left(\frac{\left[n t_{j-1}\right]}{n}\right)\right]\right]
\end{aligned}
$$

D'apròs le lemme (1.5), via la proposition (4.2), les v.a. $\sum_{j}(n)$ et $S_{n}\left(-\frac{\left[n t_{j-1}\right]}{n}\right)$ sont asymptotiquement indépendantes. Compte tenu de 1 a proposition (2.6), nous avons alors

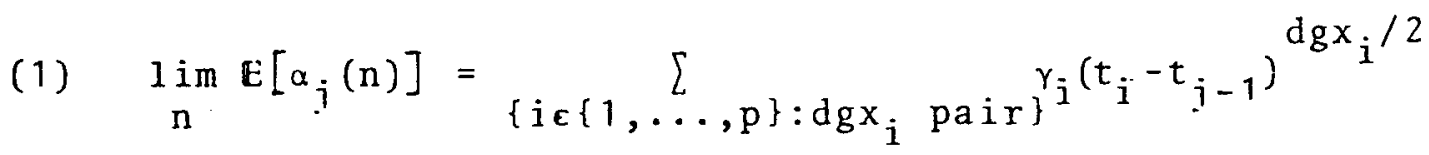

$$
\begin{aligned}
& \mathbb{E}_{\rho}\left[D_{i} f\left(x_{\infty}\left(t_{j-1}\right)\right)\right]
\end{aligned}
$$

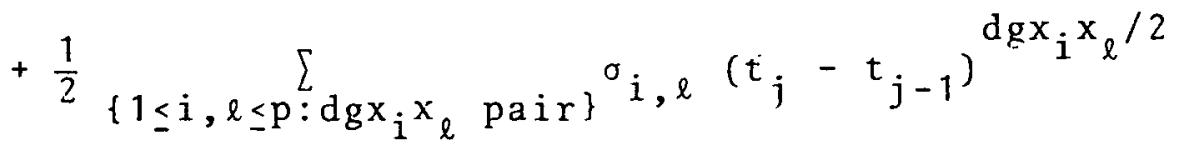

$$
\begin{aligned}
& \mathbb{E}_{\rho}\left[D_{i} D_{\ell} f\left(x_{\infty}\left(t_{j-1}\right)\right)\right],
\end{aligned}
$$

où $\quad \gamma_{i}=\frac{1}{\left(\operatorname{dgx_{i}} / 2\right) !} L_{\lambda}^{\operatorname{dg} x_{i} / 2} x_{i} \quad$ et $\quad \sigma_{i, \ell}=\frac{1}{\left(d g x_{i} x_{\ell} / 2\right) !} L_{\lambda}^{\operatorname{dg} x_{i} x_{\ell} / 2} x_{i} x_{\ell}$ D'autre part, pour $i, l \in\{1, \ldots, p\}, j \in\{1, \ldots, q\}$, posons, 


$$
\begin{array}{r}
\delta_{i, \ell}^{j}(n)=\mathbb{E}:\left[x _ { i } ( \sum _ { j } ( n ) ) x _ { \ell } ( \sum _ { j } ( n ) ) \left[D_{i} D_{\ell} f\left[s_{n}\left(\frac{\operatorname{lnt} j-1}{n}\right)\left(\theta \sum_{j}(n)\right)\right]-\right.\right. \\
-D_{i} D_{\ell} f\left(S_{n}\left(\frac{\left.\left.\left[n+\frac{j-1}{n}\right)\right)\right]}{n}\right]\right.
\end{array}
$$

Pour $\operatorname{dg} x_{i}+d g x_{2} \geq 3$, nous avons, en appliquant 1'inégalité de schwartzet le lemme (4.3), $\left|\delta_{i, \ell}^{j}(n)\right| \leq\left[2 C_{i, \ell}|| D_{i} D_{\ell} f|| \sup _{1 \leq j \leq q}\left(\frac{\left[n t_{j}\right]-\left[n t_{j-1}\right]}{n}\right)\right]\left(\frac{\left[n t_{j}\right]-[n t j-1]}{n}\right)$, où $C_{i, \ell}$ est une constante $>0$ indépendante de $n$ et de la partition $\left(t_{j}\right)_{1 \leq j \leq q}$ de $[0, t]$.

Soient $i, l \in\{1, \ldots, p\}$ avec $d g x_{i}=d g x_{\ell}=1$. Pour tout $\varepsilon>0$, il existe $\alpha>0$ tel que pour tout u $\mathrm{N}$ vérifiant $|u| \mid<\alpha$, on ait

$$
\sup _{u \in N}\left|D_{i} D_{\ell} f\left(\begin{array}{ll}
u & v
\end{array}\right)-\Gamma_{i} D_{\ell} f(u)\right|<\varepsilon
$$

Nous avons alors

$$
\begin{aligned}
& \left|\delta_{i, \ell}^{j}(n)\right| \leq \varepsilon E\left[\left|x_{i}\left(\Sigma_{j}(n)\right) x_{\ell}\left(\Sigma_{j}(n)\right)\right|\right]+2|| D_{i} D_{\ell} f||_{\infty} \\
& E\left[\left|x_{i}\left(\Sigma_{j}(n)\right) x_{\ell}\left(\Sigma_{j}(n)\right)\right| 1_{\left\{|| \Sigma_{j}(n)||>\alpha\right\}}\right] \\
& \leq \varepsilon C_{i, \ell}\left(\frac{\left[n t_{j}\right]-[n t j-1]}{n}\right)+2\left\|n_{i} D_{\ell} f\right\|_{\infty} \sqrt{\mathbb{E}_{\left[x_{i}^{2}\left(\Sigma_{j}(n)\right) x_{\ell}^{2}\left(\Sigma_{j}(n)\right)\right]}} \\
& \left(\frac{\sqrt{E\left[\left.\left\|\Sigma_{j}(n)\right\|\right|^{2}\right]}}{\alpha}\right)
\end{aligned}
$$

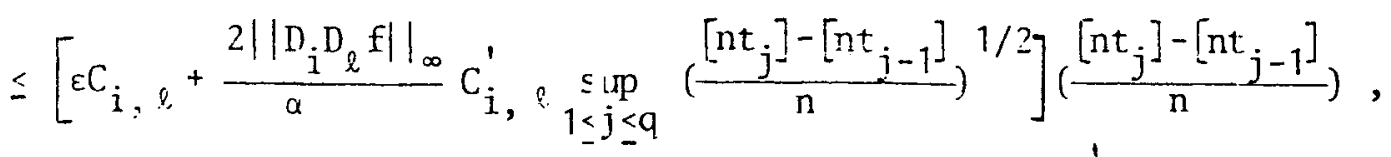


oì $C_{i, \ell}$ et $C_{i, \ell}^{\prime}$ sont des constantes $>0$ indépendantes de $n$ et de la partition $\left(t_{j}\right)_{1 \leq j \leq q}$ de $[0, t]$.

On en déduit que pour tout $\varepsilon>0$, il existe $\alpha>0$

tel que

$\left|\sum_{j=1}^{q} \beta_{j}(n)\right| \leq \sum_{j=1}^{q} \sum_{1 \leq i, \ell \leq p}\left|\delta_{i, \ell}^{j}(n)\right| \leq\left(C_{1}+\frac{C_{2}}{\alpha}\right) \sup _{1 \leq j \leq q}\left(\frac{\left[n t_{j}\right]-\left[n t_{j-1}\right]}{n}\right)^{1 / 2}+\varepsilon C_{j}$

où $\mathrm{C}_{1}, \mathrm{C}_{2}$ et $\mathrm{C}_{3}$ sont des constantes > 0 indépendantes de $\mathrm{n}$ et de la partition $\left(t_{j} ; 1 \leq j \leq q\right.$ de $[0 ; t]$.

De I'inégalité

$\left(\frac{\left[n t_{j}\right]-\left[n t_{j-1}\right]}{n}\right)^{1 / 2} \leq\left[\left(t_{j}-t_{j-1}\right)+\frac{1}{n}\right]^{1 / 2} \leq\left(t_{j}-t_{j-1}\right)^{1 / 2}+\frac{1}{\sqrt{n}}$,

il résulte que,

$\forall \varepsilon>0, \exists \alpha>0,\left|\sum_{j=1}^{q} \beta_{j}(n)\right| \leq \varepsilon C_{3}+\left(C_{1}+\frac{C_{2}}{\alpha}\right)\left(\sup _{1 \leq j \leq q}\left(t_{j}-t_{j-1}\right)^{1 / 2}+\right.$

$+\frac{1}{\sqrt{n}}$

On en déduit que, pour tout $\varepsilon>0$, il existe $\alpha>0$ tel que, pour tout entier naturel $n$,

$$
\begin{aligned}
\left|E\left[f\left(S_{n}(t)\right)-f(0)-\sum_{i=1}^{q} \alpha_{i}(n)\right]\right| & \leq \mid F\left[f\left(S_{n}(t)\right)-f\left(S_{n}\left(\frac{[n t]}{n}\right) j\right] \mid+\right. \\
& \frac{1}{2}\left|\sum_{i=1}^{p} \beta_{i}(n)\right| \\
& \leq \varepsilon C_{3}+\left(C_{1}+\frac{C_{2}}{\alpha}\right) \sup _{1 \leq j \leq q}\left(t_{j}-t_{j-1}\right)^{1 / 2}+\delta(n),
\end{aligned}
$$

où $\delta(n)$ est une suite de réels positifs, indépendante de la partition $\left(t_{j}\right)_{1 \leq j \leq q}$, convergeant vers zêro et $C_{1}, C_{2}, C_{3}$ sont des réels $>0$ indépendants de $n$ et de la partition $\left(t_{j}\right)_{1 \leq j \leq q}$ de $[0, t]$. 
En faisant tendre $n$ vers l'infini, nous voyons que :

(2) pour tout $\varepsilon>0$, il existe $\alpha>0$ tel que

$\left|\mathbb{E}\left[f\left(x_{\infty}(t)\right)\right]-f(0)-\sum_{i=1}^{q} \lim _{n} E\left[\alpha_{j}(n)\right]\right| \leq \varepsilon C_{3}+\left(C_{1}+\frac{C_{2}}{\alpha}\right) \sup _{1 \leq j \leq q}\left(t_{j}-t_{j-1}\right)^{1 / 2}$

D'après la proposition (4.2), pour toute fonction continue $h$ sur $N$, à support compact, la fonction, $t \rightarrow \mathbb{E}\left[h\left(x_{\infty}(t)\right)\right]$, est continue. De (1) il s'ensuit que, lorsque $\sup _{\substack{1 \leq j \leq q \\-q}}\left(t_{j}-t_{j-1}\right)$ tend vers zéro, $\quad \lim \sum_{j=1}^{g} E\left|\alpha_{j}(n)\right|$ tend vers $\int_{0}^{t} E_{\rho}\left[A f\left(x_{\infty}(s)\right)\right] d s$. La proposition (4.5) résulte alors de (2).

Fin de la preuve du théorème.

D'après la proposition(4.2), pour tout $t \in[0,1]$, la suite $\left\{\mathbb{E}\left[|| S_{n}(t)||^{2}\right]\right\}_{n \geq 1}$ est bornée; I'ensemble des lois des v.a. $\left\{S_{n}(t)\right\}_{n \geq 1}$ est donc relativement compact pour 1 a topologie de 1 a convergence étroite. De (4.4) et de la proposition (4.5), il résulte que la seule valeur d'adhérence de cette famille de probabilité est'la loi au temps $t$ du semi-groupe $\left(\nu_{t}\right)_{t>0}$ sur $N^{b}$ de $g . i$. A. Il s'ensuit que, pour tout $t \in[0,1], S_{n}(t)$ converge en loi vers $v_{t}$ et $1 a$ première assertion du théorème est démontrée.

$$
\text { D'autre part, des propositions (4.1) et (4.2) il résulte }
$$
que pour tout entier naturel $p$, la $v . a$.

$$
u_{n}^{b} \circ \pi_{1}\left(x_{1} \ldots x_{n}\right)-u_{n}^{b} \circ \pi_{1}\left(x_{1} \ldots x_{n-p}\right)
$$

converge vers zéro dans $\mathrm{L}^{2}$.

On en déduit que 1 a suite d'homéomorphismes $\left\{U_{n}^{b}\right\}_{n} \geq 1$ de $N$ vérifie les hypothèses de la proposition (1.2). La démonstration du 
théorème, dans ce premier cas, est donc achevée.

\section{Cas Général}

Le passage du cas précédent au cas général se fait par un procédé de truncature analogue à celui utilisé dans [5]. Nous indiquons la marche à suivre, sans entrer dans les détails.

Pour tout entier naturel $n \geq 1$, désignons par $\alpha_{n}$ l'élément de $N$ défini par:

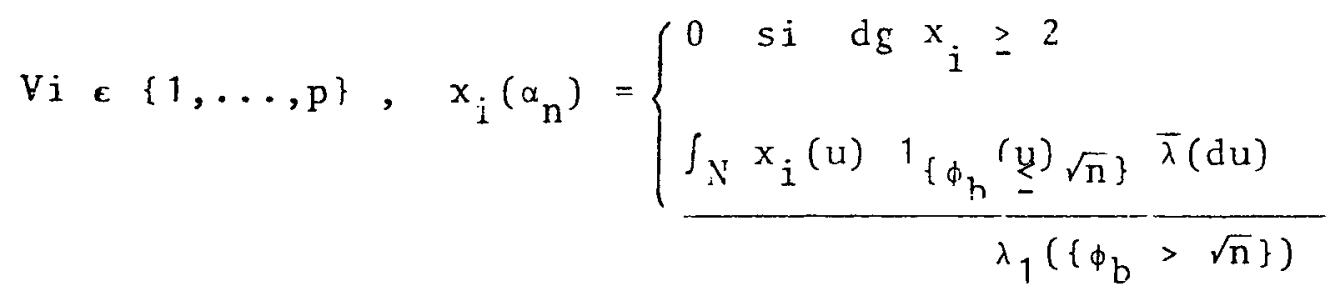
si dg $x_{i}=1$

Nous posons alors, pour $g=(u, k) \in N \times K$ et pour tout entier naturel $n \geq 1$

$$
\mathrm{T}_{n}^{\mathrm{b}}(g)=\left\{\begin{array}{lll}
(u, k) & \text { si } \phi_{b}(u) \leq \sqrt{n} \\
\left(\alpha_{n}, k\right) & \text { si } \phi_{b}(u)>\sqrt{n}
\end{array}\right.
$$

'our tout entier naturel $n \geq 1,1$ a mesure de probabilité $\mathrm{T}_{\mathrm{n}}^{\mathrm{b}}(\lambda)$ sur $\mathrm{G}$ vérifie $I^{\prime}$ hypothèse $\left.\mathrm{iii}\right)$ de $(2.1)$ et $\bar{\ell}\left(\mathrm{T}_{\mathrm{n}}^{\mathrm{b}}(\lambda)=+\infty\right.$.

Nous avons: 
(4.6) Lemme

Avec les notations précédentes,

$$
\underset{n}{\lim }|| \lambda^{n}-\left[T_{n}^{b}(\lambda)\right]^{n}||=0
$$

Preuve : On voit facilement que

$\left\|\lambda^{n}-\left[T_{n}^{b}(\lambda)\right]^{n}\right\| \leq n \lambda\left(\left\{\phi_{b}>\sqrt{n}\right\}\right) \leq \int_{G} \phi_{b}^{2}(g) 1_{\left\{\phi_{b}>\sqrt{n}\right\}}^{(g)} \lambda(d g)$,

qui tend vers zéro, quand $n$ tend vers l'infini.

(4.7) Lemme

Pour toute fonction polynome $T$ sur $N$, nous avons

$\lim \frac{1}{n d g T / 2-T} Q_{T_{n}^{b}(\lambda)} T=0$ si $\quad \mathrm{dgT} \geq 3$

$\lim _{n} \mathrm{Q}_{\mathrm{n}}^{\mathrm{b}(\lambda)} \mathrm{T}=\mathrm{Q}_{\lambda} \mathrm{T} \quad$ si $\mathrm{dg} \mathrm{T} \leq 2$

Preuve : voir lemme (4.11) de $[5]$.

Soit $T$ une fonction polynome sur $N$. Soit $\lambda$ une mesure de probabilité sur $G$ vérifiant 1 'hypothèse iii) de (2.1) et $\ell(\lambda) \geq \operatorname{dgT}$. L'expresssion $L_{\lambda}^{d g T / 2} \mathrm{~T}$ (voir $(2.5)$ ), ne dépend de $\lambda$ que par $\lambda_{2}$ et les moments.:

$\int_{N} P(u) \lambda_{1}(d u), \int_{N} P(u) \hat{\lambda}(d u), \quad P \quad \in \mathbb{C}_{1}[N] ;$ 


$$
\int_{N} P(u) \bar{\lambda}(d u), \quad P \in C_{2}[N] .
$$

Pour toute mesure de probabilité $\lambda$ sur $G$ vérifiant 1'fiypothèse iii) de (2.1) et $\ell(\lambda) \geq 2$, nous pouvons donc donner un sens à l'expression $\mathrm{L}_{\lambda}^{\mathrm{dgT} / 2 \mathrm{~T}}$, pour toute fonction polynome $\mathrm{T}$ sur $N$.

En tenant compte du lemme (4.6) et en procèdant comme pour la preuve de la proposition (2.6), on montre alors la proposition :

\section{(4.8) Proposition.}

Soit $(G, \lambda)$ vérifiant les hypothèses du théorème (2.12) . Alors pour toute fonction polynome $T$ sur $N$, la suite de fonctions polynomes $\quad\left\{\frac{1}{\mathrm{~d} g \mathrm{~T} / 2}\left[\mathrm{Q}_{\mathrm{T}_{\mathrm{n}}^{\mathrm{b}}(\lambda)}\right]^{\mathrm{n}}{ }_{\mathrm{n} \geq 1}^{\mathrm{T}}\right\}$ converge, uniformément sur tout compact de $N$, vers zérosi dgT est impair, vers la constante $\frac{1}{(\operatorname{dgT} / 2) !} \mathrm{L}_{\lambda}^{\operatorname{dgT} / 2_{\mathrm{T}}}$ si $\operatorname{dgT}$ est pair.

D'après le lemme (4.6), nous sommes amenés, pour prouver le théorème (2.12), à étudier la suite de mesures de probabilité. $\left\{U_{n} \circ \pi_{1}\left(\left[T_{n}^{b}\left(\lambda^{n}\right)\right]^{n}\right)\right\}_{n \geq 1}$. A 1 'aide du lemme (4.6) et de la proposition (4.8) on procède alors comme dans le cas étudié précédemment en remplaçant les v.a. $\left(X_{i}\right)_{i \geq 1}$ par les v.a. $\left(T_{n}^{b}\left(X_{i}\right)\right)_{i \geq 1}$. 
5. Théorème de la limite centrale pour un produit semidirect d'un groupe de Lie nilpotent simplement connexe par un groupe compact

Dans cette section, nous allons utiliser les résultats de la section 2 pour établir un théorème de la limite centrale pour un produit semi-direct d'un groupe de Lie nilpotent simplement connexe par un groupe compact.

\section{Définitions et Notations}

(5.0) Soit $G=N x_{n} K$ un produit semi-direct d'un groupe de Lie nilpotent simplement connexe $\mathrm{N}$ par un groupe compact $\mathrm{K}$.

Pour simplifier l'écriture, nous identifions $N$ et $K$ à des sous-groupes de $G$; tout élément $g$ de $G$ s'écrit donc de façon unique $g=u k$ avec $u \in N$ et $k \in K ; N$ est un sous-groupe distingué de $G$ et, pour tout couple $(u, k) \epsilon N \times K, n(k)(u)$ s'écrit $\mathrm{kuk}^{-1}$.

D'autre part, nous identifions $N$ à son algèbre de Lie $(N,[]$,$) munie du produit o défini par la formule de Campbel1-$ Hausdorff (voir (2.1)); le produit de deux éléments $g=u k$ et $g^{\prime}=u^{\prime} k^{\prime}$ de $G$ s'écrit donc $g g^{\prime}=\left[u\left(k u^{\prime} k^{-1}\right)\right] k k^{\prime}=\left[\right.$ uoAdk( $\left.\left.u^{\prime}\right)\right] k k^{\prime}$, où pour tout $k \in K$, Adk désigne l'automorphisme d'algèbre de Lie de $(N,[]$,$) (et par suite l'automorphisme de groupe de (N, 0)$ ) tangent à $1^{\prime}$ automorphisme $\mathrm{u} \rightarrow \mathrm{kuk}^{-1}$ de $N$. 
(5.1) Moments d'une mesure de probabilité sur un groupe L.C.D. compa:tement engendré $([2])$

Soit $H$ un groupe localement compact à base dénombrable, compactement engendré. Une application borélienne $\delta$ de $H$ dans $\mathbb{R}_{+}$ est appelé jauge (resp. fonction sous-additive) si elle vérifie.

$\mathrm{Fg}_{1}, \mathrm{~g}_{2}: \mathrm{H} \delta\left(\mathrm{g}_{1} \mathrm{~g}_{2}\right) \leq \delta\left(\mathrm{g}_{1}\right)+\delta\left(\mathrm{g}_{2}\right)+\mathrm{C} \quad\left(\operatorname{resp} \delta\left(\mathrm{g}_{1} \mathrm{~g}_{2}\right) \leq \delta\left(\mathrm{g}_{1}\right)+\delta\left(\mathrm{g}_{2}\right)\right)$, où $\mathrm{C}$ est une constante $>0$ indépendante de $g_{1}$ et $g_{2}$.

Une jauge $\delta$ de $H$ est dite principale s'il existe un voisinage compact $V$ de l'unité engendrant $H$ tel que $\{x: \delta(x) \leq n\} \in V^{n}, \forall n \in N^{*}$.

Sur un groupe L.C.D. compactement engendré, il existe des jauges principales et si $\delta_{0}$ est l'une d'elles, pour toute autre jauge $\varepsilon$ nous avons : $\mathrm{Vg} \in \mathrm{H} \quad \delta(\mathrm{g}) \leq \mathrm{C}_{1} \delta_{0}(\mathrm{~g})+\mathrm{C}_{2}$ où $C_{1}$ et $C_{2}$ sont des constantes $>0$ indépendantes de $g \in H$. I1 s'ensuit que si $\mu$ est une mesure de probabilité sur $H, 1$ 'expression $\int\left[\delta_{0}(\mathrm{~g})\right]^{\alpha} \mu(\mathrm{dg})<+\infty$, pour $\alpha>0$, est indépendante du choix de $\delta_{0}$; dans ce cas nous disons que $\mu$ possède un moment d'ordre $\alpha$.

(5.2) Moments d'une mesure de probabilité sur G.

Appelons $r$ la longueur de l'algèbre de Lie nilpotente. $(N,[]$,$) et désignons par$ $N^{1}=N \Rightarrow N^{2}=[N, N] \Rightarrow \ldots \Rightarrow N^{r}=\left[N, N^{r-1}\right] \Rightarrow N^{r+1}=(0)$, la suite centrale descendante de $N$. Pour tout i $\varepsilon\{1, \ldots, r\}$, désignons par $m^{i}$ un supplémentaire de $\mathrm{N}^{\mathrm{i}+1}$ dans $\mathrm{N}^{\mathrm{i}}$. Nous avons 
$N=\underset{i=1}{\oplus} m^{i} ;$ si $u \in N$, nous notons $u^{(i)}$ sa composante sur $m^{i}$. Supposons les sous-espaces $m^{i}$, $i \in\{1, \ldots r\}$, de $N$ normés par \|\| , on définit une fonction $\phi$ sur $N$ par.

$$
\phi(u)=\sup _{1 \leq i \leq r}\left\|u^{(i)}\right\|^{1 / i} \quad\left(\begin{array}{l}
u \in N) \\
1 / .
\end{array}\right.
$$

On sait ( $[5]$, lemme II.1) que, quitte à remplacer les normes données par des normes homothétiques, $\oint$ est une jauge principale sur $N$.

$$
\begin{aligned}
& \text { En posant, pour } g=(u, x) \in N \times K, \\
& \psi(g)=\sup _{k \in K} \phi(\operatorname{Adk}(u))
\end{aligned}
$$

nous obtenons une jauge principale sur $G_{i}$. Nous disons donc qu'une mesure de probabilité $\mu$ sur $G$ possède un moment d'ordre $\Leftrightarrow>0$ si

$$
\int_{G} \psi^{\alpha}(g) \mu(\mathrm{dg})<+\infty
$$

\section{(5.3) Définition.}

Soit $\left(\mathrm{J}^{\mathrm{i}}\right)_{i \geq 1}$ une suite décroissante d'idéaux de $N$ telle que $J^{1}=N$ et $J^{i}=(0)$ pour $i \geq s+1, s \in N^{*}$. Pour $i \epsilon\{0, \ldots, s\}$, notons $q_{i} 1$ a dimension de 1 'espace vectoriel $N / j_{i+1}$. Nous disons qu'une base ordonnée $\left\{e_{k}\right\}_{1 \leq k \leq p},\left(p=q_{s}\right)$, de $N$ est adaptée à la suite d'idéaux $\left(y^{i}\right)_{i \geq 1}$ si pour tout $i c\{1, \ldots, s\}$, vérifiant $q_{i} \neq q_{i-1},\left\{e_{q}+1, \ldots, e_{q_{j}}\right\}$ est une base d'un supplémentaire de $j^{i+1}$ dans $j^{i}$. 
(5.4) Soit $\lambda$ une mesure de probabilité sur G. Nous désignons par $\pi_{1}$ (resp. $\pi_{2}$ ) la projection de $G$ sur $N$ (resp. K) et nous êcrivons $\lambda_{i}=\pi_{i}(\lambda), i=1,2$. Nous notons $m$ (resp. $\stackrel{\sim}{m}$ ) la mesure de Haar normalisée du sous-groupe compact $K\left(\lambda_{2}\right)$ (resp. $\tilde{K}\left(\lambda_{2}\right)$ ) de $K$ engendré par le support, $S\left(\lambda_{2}\right)$, de $\lambda_{2}$ (resp. par $S\left(\lambda_{2}\right)\left[S\left(\lambda_{2}\right)\right]^{-1}$ ). Quitte à remplacer $G=N K$ par $G=N K\left(\lambda_{2}\right)$, nous pouvons supposer que $K\left(\lambda_{2}\right)=K$. Nous disons que $\lambda_{2}$ est apériodique si $\hat{K}^{\prime}\left(\lambda_{2}\right)=K$. Nous posons $\bar{\lambda}=\int_{K} \operatorname{Adk}\left(\lambda_{1}\right) \mathrm{m}(\mathrm{dk}) ; \bar{\lambda}$ est une mesure de probabilité sur $N$.

(5.5) Soit $\mu$ une mesure de probabilité sur $N$ possèdant un moment d'ordre 1 (i.e. $\int_{N} \phi(u) \mu(d u)<+\infty$, voir (5.2)). Appelons $\zeta$ l'application naturelle de $N$ sur $N /[N, N]$. D'après (5.2) l'intégrale $\left.\int_{N} \zeta(u)\right\lrcorner(d u)$ a un sens et défini un élément e $(\mu)$ de $N /[N, N]$. Nous disons qu'une mesure de probabilité $\mu$ sur $N$ est centrée si $\mu$ possède un moment d'ordre 1 et $\mathrm{si}$ e $(\mu)=0$.

\section{Théorème de la limite centrale dans le cas centré}

Nous supposons que la mesure de probabilité sur $N, \bar{\lambda}$, est centrée (voir $(5.4)$ et $(5.5))$.

(5.6) Notion de degré sur l'algèbre des fonctions polynomes

$$
\text { sur N }([2]) \text {. }
$$

Désignons par $\left\{e_{k}\right\} 1 \leq k \leq p$ une base de $N$ adaptée à la suite centrale descendante, $\left(N^{i}\right)_{i \geq 1}$ de $N$ (voir (5.2) et définition (5.3)); et par $\left\{x_{k}\right\}_{1 \leq k \leq p}$ le système de fonctions coordonnées associé à cette base. Nous définissons une notion de degré sur $\mathbb{C}[N]$, en attribuant 
un degré à chaque générateur $x_{k}, 1 \leq k \leq p ; 1 e$ degré de $x_{k}$, noté dg $x_{k}$, est par définition égal au plus grand entier i tel que $e_{k}$ appartienne à $\mathrm{N}^{i}$. On convient que le degré (resp. la valuation) du polynome nul est $(-\infty)$ (resp. $(+\infty)$ ). Il est facile de voir que cette notion est indépendante du choix de la base adaptée.

\section{Crochet de Lie associé à une base de $N$ adaptée à sa suite}

centrale descendante

Soit $b=\left\{e_{i}\right\}_{1 \leq i \leq p}$ une base de $N$ adaptée à sa suite centrale descendante, ' $\left(N^{i}\right)_{1 \leq i \leq r}$. Pour tout $i \in\{1, \ldots, r\}$, $\left\{e_{p_{i-1}}+1, \ldots, e_{p_{i}}\right\}$, où $p_{i}=\operatorname{dim}\left(N / N^{i+1}\right)$ et $p_{0}=0$, est une base d'un supplémentaire $\mathrm{m}^{\mathrm{i}}$ de $\mathrm{N}^{\mathrm{i}+1}$ dans $\mathrm{N}^{\mathrm{i}}$.

Pour tous $i$ et $j$ appartenant à $\{1, \ldots, r\}$, nous avons

$$
\left[m^{i}, m^{j}\right] \subset N^{i+j}=\underset{\{l: \ell \geq i+j\}}{\oplus} m^{\ell}
$$

Pour $u \in \mathrm{m}^{i}$ et $v \in \mathrm{m}^{j}$, nous notons $[\mathrm{u}, \mathrm{v}]_{\mathrm{b}}$ la composante de $[u, v]$ sur $m^{i+j}$; puis pour $u$ et $v$ appartenant à $N$, nous posons

$$
[u, v]_{b}=\sum_{1 \leq i, j \leq r}\left[u_{i}, v_{j}\right]_{b}
$$

où u $=\sum_{i=1}^{r} u_{i}$ avec $u_{i} \in m^{i}$ et $v=\sum_{j=1}^{r} v_{j}$ avec $v_{j} \in m^{j}$.

Il est clair que l'application de $N \times N$ dans $N$ qui au couple $(u, v)$ associe $[u, v]_{b}$ est bilinéaire alternée. D'autre part, pour $u \in m^{i}, v \in m^{j}, w \in m^{\ell}, \operatorname{avec} i, j, l \in\{1, \ldots, r\}$, $\left[\mathrm{u},[\mathrm{v}, \mathrm{w}]_{\mathrm{b}}\right]_{\mathrm{b}}$ n'est autre autre que la composante de $[\mathrm{u},[\mathrm{v}, \mathrm{w}]]$ sur $m^{i+j+\ell}$; il s'ensuit que $[,]_{b}$ vérifie l'identité de Jacobi. On en déduit donc que $[,]_{b}$ est un crochet de Lie sur $N$, vérifiant 
$\left[m^{i_{1}},\left[m^{i}, \ldots\left[m^{i} s-1, m^{i} s\right]_{b} \ldots\right]_{b}\right]_{b}=m^{j=j^{\hat{L}}=1^{j}}$, pour $j_{j},\{1, \ldots, r\}$, jc $\{1, \ldots, s\}$; et $\left(\mathbb{N},[,]_{b}\right)$ est une algèbre de Lie nilpotente de longueur inférieure ou égale à $r$. Nous notons ${ }^{\circ} \mathrm{b}$ le produit sur $N$ associé au crochet de Lie $[,]_{b}$ par la formule de Campbel1-Hausdorff.

(5.8) Soit $b=\left\{e_{i}\right\} 1_{\leq i \leq p}$ une base de $N$ adaptée à sa suite centrale descendante. Si $f$ est une fonction de classe $C^{1}$ sur $N$ et $v$ un élément de $N$, nous définissons

$$
n_{v} f(u)=\lim _{t \rightarrow 0} \frac{f\left(u{ }^{o} b t v\right)-f(u)}{t}
$$

Si $\lambda$ possède un moment d'ordre 2 , nous désignons par $A^{b}$ le générateur infinitésimal,

$$
A^{b}=\sum_{i=p_{1}+1}^{p_{2}}\left(L_{\lambda} x_{i}\right) D_{j}+\frac{1}{2} \sum_{1 \leq i, j \leq p_{1}}\left(L_{\lambda} x_{i} x_{j}\right) D_{i} D_{j},
$$

où $L_{\lambda}$ est défini en $(2.5)$ et $D_{i}=D_{e_{i}}$, i $c\{1, \ldots, p\}$.

Pour tout $v \in N$, le champ analytique de vecteurs tangents invariant à gauche, $D_{v}$, sur $\left(N, o_{b}\right)$, s'identifie à J'élément $v$ de l'algèbre de Lie $\left(N,[,]_{b}\right)$ de $\left(N, o_{b}\right)$.

La sous-algèbre de Lie de $(N,[]$,$) engendrée par les$ éléments $e_{i}, 1 \leq i<p_{j}$, est égale à $(N,[]$,$) . D'après la définition$ du crochet de Lie $[,]_{b}$ (voir (5.7)), il est alors clair, que la sous-algèbre de Lie de $\left(N,\left[, l_{h}\right)\right.$ engendrée par ces éléments est égale à $\left(N,[,]_{b}\right)$.

Lorsque la matrice $\left(\left(L_{\lambda} x_{i} x_{j}\right)\right)_{i, j \in\left\{1, \ldots, p_{j}\right\}}$, nous savons donc (remarque (2.13)) que le semi-groupe de convolution $\left(\nu_{t}^{b}\right)_{t>0}$ sur 
$\left(N,{ }_{b}\right)$ de g.i. $A^{b}$ est absolument continu par rapport à la mesure de Haar de $\left(N,{ }_{b}\right)$, avec une densité de classe $C^{\infty}$.

L'adaptation du lemme (2.4) à la situation précédente, nous dit que la matrice $\left(\left(L_{\lambda} x_{i} x_{j}\right)\right)_{i, j \in\left\{1, \ldots, p_{1}\right\}}$ est définie positive si et seulement si. la mesure $\lambda$ n'est pas portée par un sous-groupe fermé de $G$ de 1 a forme $N_{0}\left(a K^{-1} a\right)$ où : $N_{0}^{\prime}$ est un idéal propre de $(N,[]),$,$K -invariant (i.e. Adk \left(N_{0}\right) \subset N_{0}$, $Y k \in K$ ), contenant $[N, N]$; et a est un élément de $N$ vérifiant

(1) $x_{i}(a)=\left(\left[I-Q_{\lambda_{2}}\left(I-Q_{m}\right)\right]^{-1} x_{i}\right)\left(\int_{N} u \lambda_{1}(d u)\right), H_{i} \in\left\{1, \ldots, p_{1}\right\}$.

[On notera que des propriétés de $N_{0}$, il résulte que le groupe $N_{0}\left(a k^{-1} a\right)$ est le même pour tous les éléments a de $N$ vérifiant (1)].

Si $\lambda=\lambda_{1} \otimes \lambda_{2}$ ou $\lambda_{1}$ est centrée, on peut prendre pour a l'élément neutre de $G$.

Nous avons alors:

\section{Théorème.}

Soit $\lambda$ une mesure de probabilité sur $G$ possèdant un moment d'ordre 2 et telle que $\bar{\lambda}$ soit centrée. Soit $b=\left\{e_{i}\right\}_{1 \leq i \leq p}$ une base de $N$, adaptée à sa suite centrale descendante. Pour tout $(\mathrm{u}, \mathrm{k}) \epsilon \mathrm{N} \times \mathrm{K}$ et pour tout $\mathrm{k}_{0} \epsilon \mathrm{K}$, posons

$$
v_{n}^{b, k^{0}}(u, k)=\left(\sum_{i=1}^{p} \frac{x_{j}(u)}{d_{n} x_{i} / 2} e_{i}, \quad k_{0}^{-n} k\right) \text {. }
$$

Alors, pour tout $k_{0} \in S\left(\lambda_{2}\right)$, la suite de mesure de probabilité sur $N \times k,\left\{V^{b, k_{0}}\left(\lambda^{n}\right)\right\} n \geq 1$ converge vaguement rers la mesure de probabilité produit $v_{1}^{b} \stackrel{n}{m}$. En particulier lorsque $\lambda_{2}$ 
est apériodique, $\left\{v_{n}^{b, e}\left(\lambda^{n}\right)\right\}_{n \geq 1}$, converge vaguement vers $\nu_{1}^{b} \otimes m$.

Preuve du théorème (5.9)

Pour la notion de degré sur $\mathbf{C}[\mathrm{N}]$ définie en (5.6), le couple $(G, \lambda)$ vérifie les hypothèses de (2.1) : compte tenu de 1a formule de Campbel1-Hausdorff, l'hypothèse i) (resp. ii)) est une conséquence des relations $\left[\mathrm{N}^{\mathrm{i}}, \mathrm{N}^{\mathrm{j}}\right] \subset \mathrm{N}^{\mathrm{i}+\mathrm{j}}, \mathrm{Hi}, \mathrm{j} \geq 1$, (resp. du fait que les $N^{i}, i \geq 1$, sont stables par les éléments Adk, $k \in K$ ) ; enfin 1 'hypothèse iij) signifie que $\bar{\lambda}$ est centrée.

lin remarquant d'une part que la condition (*) de (2.11) équivaut à l'existence d'un moment d'ordre 2 pour $\lambda$ et d'autre part que les groupes $\left(N,{ }^{\circ} \mathrm{b}\right)$ et $\mathrm{N}^{\mathrm{b}}$ associés à $\mathrm{b}$ respectivement en (5.7) et (2.9) coincident, nous royons que le théorème (5.9) résulte du théorème $(2.12)$.

\section{Théorème de la limite centrale dans le cas général}

$\lambda$ est une mesure de probabilité sur $G$ possèdant un moment d'ordre 1 .

(5.10) Suite graduée d'idéaux de $N$ associée à $\lambda$.

Considérons 1 'ensemble

$E=\{(\ell, k)$ c $\mathbb{N} \times \mathbb{N}: 0 \leq k<\ell\} \cup\{(1,1)\}$ muni de la relation d'ordre total $\geq$ définie par

$(\ell, k) \geq\left(\ell^{\prime}, k^{\prime}\right) \Leftrightarrow\left\{\begin{array}{l}\ell>\ell^{\prime} \\ \text { ou } \\ \ell=\ell^{\prime} \text { et } k \geq k^{\prime} .\end{array}\right.$

On vérifie aisément que l'ordre ainsi défini sur E est celui qui est induit par la bijection : 


$$
\begin{aligned}
& \text { c: } E \rightarrow N^{*} \\
& (\ell, k) \rightarrow \sigma(\ell, k)= \begin{cases}\ell(\ell-1) / 2+k+2 & \text { si } \ell \geq 2 \\
k+1 & \text { si } \ell=1\end{cases}
\end{aligned}
$$

Nous appelons suite graduée d'idéaux de $N$ associée à $\lambda$, la suite décroissante d'idéaux de $N$ définie de la façon suivante: $J^{1,0}(\lambda)=N^{\prime}, J^{1,1}(\lambda)=\zeta^{-1}(e(\bar{\lambda}))$ et $J^{\ell, k}(\lambda),(\ell, k) \in F$ avec l. $\geq 2$, est $l^{\prime}$ idéal de $x^{\ell}$ formé des éléments de $N^{\ell}$ qui s'écrivent $\left[\mathrm{u}_{1},\left[\mathrm{u}_{2}, \ldots,\left[\mathrm{u}_{\ell-1}, \mathrm{u}_{\ell}\right] \ldots\right], \mathrm{u}_{1}, \ldots, \mathrm{u}_{\ell} \in \mathrm{N}\right.$, où au moins $\mathrm{k}$ éléments parmi les $u_{i}, \quad i \in\{1, \ldots, \ell\}$, appartiennent à $\tau^{-1}(e(\bar{\lambda}))$. Nous avons $y^{\ell, 0}(\lambda)=N^{\ell}, \forall \ell \geq 1 ; j^{\ell, k}(\lambda)=0$ si $\ell>r$ et $\left[g^{\ell, k}(\lambda), j^{\ell^{\prime}, k^{\prime}}(\lambda)\right]=j^{\ell+\ell^{\prime}, k^{+}+k^{\prime}(\lambda)}$ pour $(\ell, k)$ et $\left(\ell^{\prime}, k^{\prime}\right) \in E$.

\section{(5.11) Notion de "degré suirant $\lambda$ "sur C [ $]$.}

Désignons par $\left\{e_{k}\right\}_{1 \leq k \leq p}$ une base de $N$ adaptée à 1 a suite graduée d'idéaux de $N$ associée à $\lambda$ (voir définitions (5.3) et $(5.10))$; et par $\left\{x_{k}\right\}_{1 \leq k \leq p}$ le système de fonctions coordonnées associée à cette base. Nous dêfinissons une notion de dégré sur $\mathbb{C}[\mathrm{N}]$ en attribuant un dégré à chaque générateur $x_{k}, 1 \leq k \leq p ; 1$ de degré $\underline{\text { suivant } \lambda}$ de $x_{k}$, noté $\mathrm{dg}^{\lambda} \mathrm{x}_{\mathrm{k}}$, est par définition égal à 1 si $e_{k} n^{\prime}$ appartient pas à $N^{2}$, égal à $\sup \left\{s+\ell:(s, l) \in E, e_{k} \in y^{s, l}(\lambda)\right\}$ si $e_{k}$ appartient à $N^{2}$. On convient que le degré (resp. la valuation) suivant $\lambda$ du polynome nul est $(-\infty)$ (resp. $(+\infty))$. Il est facile de voir que cette notion de degré sur $\mathbb{C}[\mathrm{A}]$ est indépendante du choix de la base de $N$ adaptée à la suite graduée d'idéaux associée à $\lambda$.

Dans le cas où la mesure de probabilité $\bar{\lambda}$ est centrée, $1 \mathrm{a}$ suite graduée d'idéaux associée à $\lambda$ n'est autre que la suite centrale descendante de $N$; nous retrouvons alors la notion ce cegré 
définie en (5.6).

(5.12) Crochets de Lie associés à une hase de $N$ adaptée à \{\}$\left.^{\ell, k}(\lambda),(\ell, k) \in E\right\}$.

Soit $b=\left\{e_{i}\right\} 1 \leq i \leq p \quad$ une base de $N$ adaptée à la suite d'idéaux $\left\{g^{\ell, k}(\lambda),(\ell, k) \in E\right\}$. Pour tout élément $(\ell, k)$ de $E$, posons

$$
q_{\sigma(\ell, k)-1}=\operatorname{dim} N / J^{\ell, k}(\lambda) \quad \text { (roir la définition de } \sigma \text { en }
$$

si $\bar{\lambda}$ est centrée $q_{2}=q_{1}$, si $\bar{\lambda}$ n'est pas centrée $q_{2}=q_{1}+1$.

Pour tout élément $(l, k)$ de $E$ vérifiant $q_{\sigma(l, k)-1} \neq q_{\sigma(l, k)}$, $\left\{\mathrm{e}_{\sigma(\ell, k)-1}+1, \ldots, \mathrm{e}_{\mathrm{q}_{\sigma(\ell, k)}}\right\}$ est une base d'un supplémentaire $m^{\ell, k}$ de $\jmath^{\ell} 0, k^{k}(\lambda)$ dans $g^{\ell, k}(\lambda)$, où $\left(\ell_{0}, k_{0}\right)=\sigma^{-1}(\sigma(l, k)+1)$.

Pour tous couples $(l, k)$ et $\left(\ell^{\prime}, k^{\prime}\right)$ de $E$, nous avons

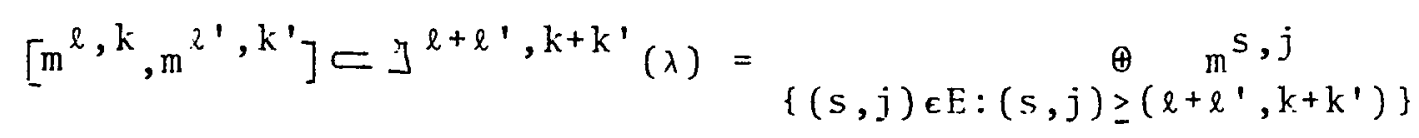

Pour $u \in \mathrm{m}^{\ell, k}$ et $\mathrm{v} \in \mathrm{m}^{\ell^{\prime}, \mathrm{k}^{\prime}}$, nous notons $[\mathrm{u}, \mathrm{v}]_{\mathrm{b}}$ la composante de $[u, v]$ sur $m^{\ell+l^{\prime}, k^{k+k^{\prime}}}$. Si $u \in N$, pour tout couple $(\ell, k)$ de $E$, nous notons $u^{(\ell, k)}$ la composante de $u$ sur $m^{\ell, k}$ nous avons

$$
\mathrm{u}=\sum_{(\ell, \mathrm{k}) \epsilon E} \mathrm{u}^{(\ell, \mathrm{k})}=\sum_{\{(\ell, \mathrm{k}) \in E: \ell \leq \mathrm{r}\}} \mathrm{u}^{(\ell, \mathrm{k})}
$$

Pour $u$ et $v$ appartenant à $l$, posons alors 


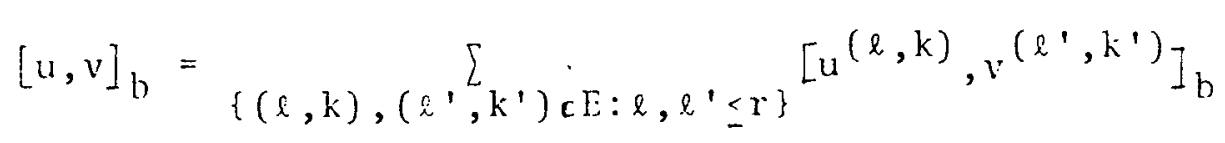

Il est clair que l'application de $N \times N$ dans $N$ qui au couple $(u, v)$ associe $[u, v]_{b}$ est bilinéaire alternée. D'autre part, pour $u \in m^{\ell, k}, v \in m^{\ell^{\prime} k^{\prime}}$, we $\mathrm{m}^{\ell "}, k^{\prime \prime}$, avec $(l, k),\left(l^{\prime}, k^{\prime}\right)$, $\left(\ell^{\prime \prime}, k^{\prime \prime}\right) \in E,\left[u,[v, w]_{b}\right]_{b}$ n'est autre que la composante de $\left[u,[v, w]\right.$ sur $m^{\ell+\ell^{\prime}+\ell ", k^{\prime}+k^{\prime}+k^{\prime \prime}} ;$ il s'ensuit que $[,]_{b}$ vérifie l'jdentité de Jacobi. On en déduit que $\left[, I_{b}\right.$ est un crochet de Lie de $N^{\prime}$, vérifiant

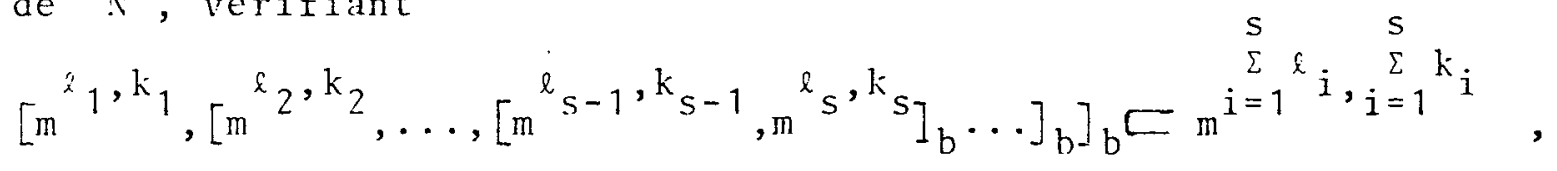
pour $\left(\ell_{i}, k_{i}\right) \in E, i \in\{1, \ldots, s\}$; et $\left(N,[,]_{b}\right)$ est une algèbre de Lie nilpotente de longueur inférieure ou égale à $r$. Nous notons ${ }^{\circ} b$ le produit sur $N$ associé au crochet de Lie $[,]_{b}$ par la formule de Campbe11-Hausdorff.

D'autre part, nous définissons un nouveau crochet de Lie $[,]_{b} \operatorname{sur} N$ en posant, pour $u$ et $v \in N$,

$$
[u, v]_{b}^{\prime}=\left[u-u^{(1,1)}, v-v(1,1)\right]_{b} .
$$

Nous notons ob le produit sur $N$ associé à ce nouveau crochet de Lie par 1a formule de Campbel1-Hausdorff. Nous avons

$$
\left.u \cdot{ }^{\prime} b^{v}=\left(u-u^{(1,1)}\right){ }^{o} b^{(v-v}(1,1)\right)+u^{(1,1)}+v^{(1,1)} \text {, }
$$

pour $u, r \in N$.

Nous notons ad(resp. ad ${ }^{b}$ et ad, $\left.{ }^{b}\right)$ la représentation adjointe de 1 'algèbre de Lie $(N,[]),\left(r e s p \cdot\left(N,[,]_{b}\right)\right.$ et $\left(N,[,]_{b}\right)$ ). 
(5.13) Soit $b=\left\{e_{i}\right\}_{1 \leq i \leq p}$ la base de $x$ considérée en (5.12). Dans le cas où $\bar{\lambda}$ n'est pas centrée, posons

$$
f=\int_{K} \operatorname{Adk}\left(e_{q_{2}}\right) m(d k) ;
$$

$f$ est un élément K-invariant de $N$ et $f-\mathrm{e}_{\mathrm{q}_{2}} \in[\mathrm{N}, \mathrm{N}]$. Nous désignons par $\bar{b}$ la base de $N^{\prime}$ qui se déduit de $\mathrm{b}$ en remplaçant $\mathrm{e}_{\mathrm{q}_{2}}$ par f.

$$
\begin{aligned}
& \text { Désignons par }{ }^{\tau} \mathrm{b} \text { l'lément de } N \text { défini par } \\
& \tau_{\mathrm{b}}=\left\{\begin{array}{lll}
{\left[\int_{N} x_{q_{2}}(u) \cdot \bar{\lambda}(d u)\right] f} & \text { si } \bar{\lambda} \text { n'est pas centrée } \\
0 & \text { sinon }
\end{array}\right.
\end{aligned}
$$

${ }^{\tau} \mathrm{b}$ est un élément K-invariant de $\mathrm{N}$.

Nous munissons 1 'espace $N \times \mathbb{R}$, (resp. $N \times K \times R$ ), du produit, noté ob́, (resp. noté multiplicatirement), défini par

$$
\begin{aligned}
& \left.(u, t) o_{b}^{\prime}(v, s)=\underset{b}{\left(u_{\circ}^{\prime}\right.} \operatorname{Exp} a d^{\bar{b}} t_{\tau} b(v), t+s\right) \text { pour } u, v \in N \text { et } \\
& s, t \in \mathbb{R} \text {, } \\
& \text { [resp. }(u, k, t)\left(v, k^{\prime}, s\right)=\left(u_{\circ} b^{\prime} \operatorname{Expad}^{\bar{b}} t_{T}(\operatorname{Adk}(v)), k k^{\prime}, t+s\right) \\
& =\left(u \circ b_{b}^{\prime} \text { Ad } k\left(\operatorname{Exp} a d^{\bar{b}} t_{t}(v)\right), k k^{\prime}, t+s\right),
\end{aligned}
$$

(car ${ }^{\top} b$ est $K$-invariant), pour $u, r^{\prime} \in N^{\prime}, k, k^{\prime} \epsilon k^{\prime}$ et $\left.t, s \in \mathbb{R}\right]$.

Nous notons $M(b)$ (resp. H(b)) les groupes ainsi obtenus. $M(b)$ est un groupe de Lie nilpotent simplement connexe qui est un produit semi-direct de $\left(N,{ }_{b}^{\prime}\right)$ par $(\mathbb{R},+)$. $H(b)$ est un produit semidirect de $\left(N,{ }^{\circ} b^{\prime}\right)$ par le produit direct des groupes $K$ et $(R,+)$; c'est donc aussi le produit semi-direct du groupe de Lie nilpotent simplement connexe $M(b)$ par le groupe compact $K$. 
(5.14) Soit $b=\left\{e_{i}\right\}_{1 \leq i \leq p}$ la base de $N$ considérée en $(5.12)$ et $(5.15)$. Si $f$ est une fonction de classe $C^{1}$ sur $x \times \mathbb{R}$ et $z$ est un élément de $N \times R$ nous posons

$$
D_{z} f(y)=\lim _{t \rightarrow 0} \frac{f\left(y_{b}^{\prime} t_{z}\right)-f(y)}{t}
$$

pour $y \in N^{\prime} \times \mathbb{R}$.

Désignons par $\lambda^{\mathrm{b}}$ la mesure de probabilité produit $\left[\lambda * \varepsilon_{\tau_{b}}-1\right] \otimes \varepsilon_{1}$ sur $G \times \mathbb{R}$. Lorsque $\lambda$ possède un moment d'ordre 2 sur $G, \lambda^{b}$ possède un moment d'ordre 2 sur $H(b)$. Nous considérons alors le générateur infinitésimal,

$$
\begin{array}{r}
A^{b}=\sum_{i=q_{2}+1}^{q_{3}}\left(L_{\lambda} b x_{i}\right) D_{\left(e_{i}, 0\right)}+\frac{1}{2} \sum_{1 \leq i, j \leq q_{2}}\left(L_{\lambda} b x_{i} x_{j}\right) D_{\left(e_{i}, 0\right)}{ }^{D}\left(e_{j}, 0\right) \\
+D_{(0,1)},
\end{array}
$$

où $L_{\lambda} b$ est défini en $(2.5)$.

Définissons sur $N \times \mathbb{R}$ le crochet de Lie suivant :

$[(u, t),(v, s)]_{b}=\left([u, v]_{b}^{\prime}+t\left[\tau_{b}, v\right]_{\bar{b}}-s\left[\tau_{b}, u\right]_{\bar{b}}, 0\right)$, pour $u, v_{\varepsilon} N$ et $t, s \in \mathbb{R}$, où $\bar{b}$ désigne $1 \mathrm{a}$ base de $\mathrm{N}$ qui se déduit de $b$ en remplaçant $e_{q_{2}}$ par $f=\int_{K} \operatorname{Adk}\left(e_{q_{2}}\right) m(d k)$, dans le cas où $\bar{\lambda}$ n'est pas centrée et $\bar{b}=b$ si $\bar{\lambda}$ est centrée. L'algèbre de Lie du groupe $M(b)$ est alors $\left(N \times \mathbb{R},[,]_{b}\right)$. Notons $\exp _{M}(b)$ l'application exponentielle du groupe $M(b)$; pour tout élément $z$ de $\Lambda \times \mathbb{R}$, le champ analytique de vecteurs tangents invariant à gauche $\mathrm{D}_{z}$ sur $M(b)$ s'identifie à l'élément $\exp _{M(b)^{2}}^{-1}$ de $I^{\prime}$ algèbre de lie $\left(N \times \mathbb{R},[,]_{b}\right)$ de $M(b)$; en particulier si $z$ est de la forme $(u, 0)$ avec $u \in N$ ou $(0, t)$ arrec $t \in \mathbb{R}, D_{z} \quad s^{\prime}$ identifie à $z$. 
Dans le cas où la matrice $\left(\left(L_{\lambda} b^{x_{i}} x_{j}\right)\right)_{1 \leq j, j \leq q_{2}}$ est définie positive, on voit alors que 1 'on a (avec les notations de 1 a remarque $(2.15))$,

$$
\check{d}_{1}\left(A^{b}\right)=N \times \mathbb{R} \quad \text { et } \quad y_{2}\left(A^{b}\right)=N \text {. }
$$

Si bien que le semi-groupe de convolution $\left(\nu_{t}^{b}\right)_{t>0}$ sur $M(b)$ de g.i. $A^{b}$, s'écrit $\left(\mu_{t}^{b} \otimes \varepsilon_{t}\right)_{t>0}$ où $\mu_{t}^{b}$ est absolument continue par rapport à la mesure de Haar de $N$ avec une densité de classe $C^{\infty}$.

L'adaptation du lemme (2.14) à la situation présente, nous dit que la matrice précédente est définie positive si et seulement si la mesure $\lambda$ n'est pas portée par la classe de ${ }^{\top} b$ modulo un sousespace fermé de $\mathrm{G}$ de 1 a forme $\mathrm{N}_{0}\left(\mathrm{aKa}{ }^{-1}\right)$, où : $N_{0}$ est un idéal propre de $(\mathrm{N},[]),, \mathrm{K}$-invariant, contenant $[\mathrm{N}, \mathrm{N}] ;$ et a est un élément de $N$ vérifiant

(1) $x_{i}(a)=\left(I-Q_{2}\left(I-Q_{m}\right)-1 x_{i}\right)\left(\int_{N} u \lambda_{1} * \varepsilon_{\tau_{b}-1}(d u)\right) \quad v i \in\left\{1, \ldots, q_{2}\right\}$ [On notera que des propriétés de $N_{0}$, il résulte que, pour tous éléments $b$ et $c$ de $N, \quad b N_{0}^{\prime} K c=b^{\prime} N_{0} K c^{\prime}=N_{0} b^{\prime} K c^{\prime}$, où

$$
x_{i}\left(b^{\prime}\right)=x_{i}(b) \text { et } x_{i}\left(c^{\prime}\right)=x_{i}(c) \text { Yi } \in\left\{1, \ldots, q_{2}\right\}
$$

et

$$
x_{i}\left(b^{\prime}\right)=x_{i}\left(c^{\prime}\right)=0 \quad \text { Hi } \in\left\{q_{2}+1, \ldots, p\right\}
$$

Si bien que d'une part le groupe $N_{0}\left(a \mathrm{Ka}^{-1}\right)$ est le même pour tous les éléments a de $N$ vérifiant (1) et d'autre part $\left.\tau_{b} N_{0}\left(a \mathrm{Ka}^{-1}\right)=N_{0}\left(a \mathrm{Ka}^{-1}\right) \tau_{b}\right]$.

Si $\lambda_{1} * \varepsilon_{\mathrm{t}^{-1}}$ est centrée ou si $\lambda=\lambda_{1} \otimes \lambda_{2}^{\prime}$, on peut prendre pour a l'élément neutre e de G. 


\section{(5.15) Théorème}

Soit $\lambda$ une mesure de probabilité sur $G$ possèdant un moment d'ordre 2. Soit $b=\left\{e_{i}\right\} 1 \leq i \leq p$ une base de $N$ adaptée à 1 a suite graduée d'idéaux de $N$ associée à $\lambda$ (voir (5.10)). Pour tout $(u, k) \in N \times K$ et pour tout $k_{0} \in K$, posons

$$
v_{n}^{b, k_{0}}(u, k)=\left(\sum_{i=1}^{p} \frac{x_{i}\left(u o\left(-n \tau_{b}\right)\right)}{d g^{\lambda} x_{i} / 2} e_{i}, k_{0}^{-n} k\right) .
$$

Alors, pour tout $k_{0} \in S\left(\dot{\lambda}_{2}\right)$, 1a suite de mesures de probabilité sur $N \times K, \quad\left\{V_{n}^{b, k_{0}}\left(\lambda^{n}\right)\right\}_{n \geq 1}$ converge vaguement vers la mesure de probabilité produit $\mu_{1}^{b} \underset{m}{m}$. En particulier, lorsque $\lambda_{2}$ est apériodique, $\left\{V_{n}^{b, e}\left(\lambda^{n}\right)\right\}_{n \geq 1}$ converge vaguement vers $\mu_{1}^{b} \otimes m$.

\section{Preuve :}

Munissons l'espace $N \times \mathbb{R}$ (resp. $G \times \mathbb{R}=N \times K \times \mathbb{R}$ ) $d u$ produit, noté o (resp. multiplicativement), défini par

$$
(u, t) \circ(v, s)=\left(u \circ \operatorname{Exp} \operatorname{adt} \tau_{b}(v), t+s\right),
$$

pour $u, v \in N$ et $s, t \in R$.

[resp. $(u, k, t)\left(v, k^{\prime}, s\right)=\left(u_{\circ} \operatorname{Exp} \operatorname{adt} \tau_{b}(\operatorname{Adk}(v)), k^{\prime}, t+s\right)$

$$
=\left(u \circ \operatorname{Adk}\left(\operatorname{Exp} \operatorname{adt} \tau_{b}(v)\right), k^{\prime}, t+s\right),
$$

pour $u, v \in N^{\prime}, k, k^{\prime} \in K$ et $\left.s, t \in R\right)$.

Nous notons $M$ et $H$ les groupes ainsi obtenus. $M$ est un groupe de Lie nilpotent simplement connexe qui est un produit semidirect de $\left(\mathrm{N}, 0^{\circ}\right)$ par $(\mathbb{R},+)$. H est un produit semi-direct de $(N, 0)$ par le produit direct des groupes $K$ et $(R,+)$; c'est donc aussi le produit semi-direct du groupe de Lie nilpotent 'simplement connexe $M$ par le groupe compact $K$. 
Soit $\left(x_{j}\right)_{i \geq 1}$ une suite de v.a. à valeurs dans $G$, indépendantes et de $1 \mathrm{ci}$ commune $\lambda$. Ecrivons $X_{i}=Y_{i} k_{i}, i \geq 1$, où $Y_{i}$ et $k_{i}$ sont des v.a. à valeurs respectivement dans $N$ et $k$. En tenant compte que l'élément de $N$, ${ }^{\top} b$, est K-invariant, le produit $x_{1} \ldots x_{n} \tau_{b}^{-n}=x_{1} \ldots x_{n}\left(-n \tau_{b}\right)$ s'écrit alors $N_{n} k_{n}$ avec $N_{n}=z_{1} \circ\left[\tau_{b} \circ \operatorname{Adk}_{1}\left(z_{1}\right) \circ\left(-\tau_{b}\right)\right] \circ \ldots \circ\left[(n-1) \tau_{b} \circ \operatorname{Adk} k_{1} \ldots k_{n-1}\left(z_{n}\right) \circ\right.$ $\left.\left(-(n-1) \tau_{b}\right)\right]$

$=z_{1} \circ \operatorname{Exp} a d \tau_{b}\left(\operatorname{Ak}_{1}\left(Z_{2}\right) \circ \ldots \operatorname{Exp} \operatorname{ad}(n-1) \tau_{b}\left(\operatorname{Adk}_{1} \ldots k_{n-1}\left(z_{n}\right)\right)\right.$, en posant $Z_{i}=Y_{i} \circ\left(-\tau_{b}\right), i \geq 1$, et $\quad k_{n}=k_{1} \ldots k_{n}$.

Pour tout entier naturel $n \geq 1$, nous voyons donc que $\left(N_{n}, K_{n}, n\right)$ est égal au produit dans $H$ des v.a. $\left(Z_{i}, k_{i}, 1\right)$, $1 \leq \mathrm{i} \leq \mathrm{n}$, à valeurs dans $N \times K \times \mathbb{R}$, indépendantes et de loi commune $\lambda^{\mathrm{b}}$.

Nous définissons une notion de degré sur $\mathbb{C}[M]=\mathbb{C}[N x \mathbb{R}]$, en attribuant un degré à tout élêment $T$ de $\mathbb{C}[N \times R]$ de 1 a forme $\mathrm{T}_{1} \otimes \mathrm{T}_{2}$ où $\mathrm{T}_{1} \in \mathbb{C}[\mathrm{N}]$ et $\mathrm{T}_{2} \in \mathbb{C}[\mathbb{R}] ; 1$ e degré de $\mathrm{T}$ est par définition égal à $\mathrm{dg}^{\lambda} \mathrm{T}_{1}+2 \mathrm{dg} \mathrm{T}_{2}$, où $\mathrm{dg}^{\lambda} \mathrm{T}_{1}$ est défini en (5.11) et $\mathrm{dg} \mathrm{T}_{2}$ est le degré habituel d'un élément $\mathrm{T}_{2}$ de $\mathbb{C}[\mathbb{R}]$.

Pour cette notion de degré sur $\mathbb{C}[M]$, le couple $\left(\mathrm{H}, \lambda^{b}\right)$ vérifie les hypothèses de (2.1): 1'hypothèse i) est une conséquence des relations $\left.\left[J^{\ell, k}(\lambda), J^{\ell^{\prime}, k^{\prime}}(\lambda)\right] \subset\right]^{\ell+\ell, k^{+k^{\prime}}(\lambda), \quad Y(l, k)}$ et $\left(\ell^{\prime}, k^{\prime}\right)$ c $\left.E ; 1^{\prime h y p o t h e ̀ s e ~} i i\right)$ résulte du fait que les idéaux $J^{l, k}(\lambda)$ de $N,(\ell, k) \epsilon E$, sont stables par les éléments Adk; k $\epsilon$; enfin l'hypothèse iii) signifie que $\bar{\lambda} * \varepsilon_{\tau_{b}}^{-1}$ est centrée sur $N$. 
puisque la mesure de probabilité $\lambda$ possède un moment d'ordre 2 sur $G$, il en est de même de 1 a mesure $\lambda^{b}$ sur $H ; \lambda^{b}$ vérifie alors 1 a condition (*) de (2.11). En remarquant alors que le groupe $M^{b^{\prime}}$ associée à $1 a$ base $b^{\prime}=\left\{\left(e_{i}, 0\right): 1 \leq i \leq p\right\} \cup\{(0,1)\}$ de l'espace vectoriel $N \times \mathbb{R}$ (voir (2.9)) coincide avec le groupe $M(b)$ construit en (5.13), le théorème (5.15) résulte du théorème (2.12) . 
$1 \mathrm{i}$ - Théorème do la limite centrale pour un produit semi-direct d'un groupe de Lie résoluble simplement conncxe de type rigide par un groupe compact

Soit $R$ un groupe de Lie résoluble connexe ayant $(R,[]$, pour algèbre de Lie. Désignons par Ad la représentation adjointe de $R$; d'après le théorème de Lie nous savons qu'il existe une base de $R$ qui triangularise simultanément tous les éléments de AdR. Nous disons que $R$ est de type rigide si les valeurs propres des éléments de AdR sont toutes de module égal à 1 .

Nous avons :

(6.1) Théorème

Soit $R$ un groupe de Lie résoluble connexe. Désignons par $D(R) \quad l$ lensemble des parties semi-simples des éléments de AdR; $D(R)$ est un groupe de Lie connexe abélien qui est en outre compact si $R$ est de type rigide. On munit alors l'espace produit $R \times D(R)$ d'un produit - explicitement décrit tel que :

i) $(R, \cdot)$ est un groupe de Lie nilpotent connexe et $(R \times D(R), \cdot)$ est le produit semi-direct du groupe $(R, \cdot)$ et du groupe de Lie abélien connexe $D(R)$.

ii) I'application $R \rightarrow(R \times D(R), \cdot)$, où $D(r)$

$$
r \rightarrow(r, D(r))
$$

désigne la partie semi-simple de Adr, est un isomorphisme de groupes 
de Lie de $R$ sur son image.

\section{Preuve du théorème.}

Choisissons une sous-algèbre de Cartan $९$ de $\mathcal{S}$ (i.e. une sous-algèbre nilpotente de $R$ qui est son propre normalisateur). Désignons par ad 1 a représentation adjointe de $R$. Pour toute forme linéaire complexe a sur $\beta$, posons

$$
\mathbb{B}_{\alpha}=\left\{X \in \mathcal{X}_{\mathbb{C}}^{\mathbb{C}}: \exists \ell \in \mathbb{N} /(\operatorname{adP}-\alpha(P) I)^{\ell}(X)=0 \quad \text { HP } \in \text { }\right\}
$$

Notons $\Delta$ l'ensemble des formes linéaires $\alpha$ telles que

$$
R_{\alpha} \neq(0) \text {. }
$$

Nous avons

$$
3=B_{0}, R^{C}=\underset{\alpha \in \Delta}{\oplus} \dot{R}_{\alpha}
$$

et

$$
\left[\beta_{\alpha}, \beta_{\beta}\right] \subset \beta_{\alpha+\beta} \quad, \quad \forall_{\alpha}, \beta \in \Delta
$$

Si $u \in R$, nous notons $u_{a}$ sa composante sur $\Re_{\alpha}, \alpha \in \Delta ;$ nous avons $\mathrm{u}=\sum_{\alpha \in \Delta} \mathrm{u}_{\alpha} \cdot$ Posons

$$
\alpha(u)=\alpha\left(u_{0}\right), u \in R, \alpha \in \Delta ;
$$

nous savons ([6]) que:

1) a est une forme linéaire sur $\mathcal{X}$ nulle sur le nilradical $\mathcal{N}$ de $R$ (i.e. le plus grand idéal nilpotent de $\mathcal{S}$ ); et tout élément $u$ de $\mathcal{K}$ tel que $\alpha(u)=0 \quad \forall \alpha \in \Delta$, appartient à $\mathcal{P}^{P}$.

2) Tout élément $\alpha$ de $\Delta$ est la différentielle en e d'un homomorphisme $\phi_{\alpha}$ de $\mathbb{R}$ dans $\left(C^{*}, x\right)$. Pour tout $\alpha \epsilon \Delta, \phi_{\alpha}$ prend la valeur 1 sur le nilradical $N$ de $R$ (i.e. le plus grand sousgroupe distingué nilpotent de $R$ ) ; et tout élément $r$ 'de $R$ pour lequel $\phi_{\alpha}(r)=1 \quad V_{\alpha} \in \Delta$, appartient à $\mathrm{N}$. 
[D'après le théorème de Lic, nous pouvons trouver une base de $R$ qui triangularise simultanément les éléments de AdR; alors la famille $\left\{\phi_{0}, \alpha \in \Delta\right\}$ n'est autre que la famille d'homomorphismes définie par les êléments diagonaux].

$$
\text { Pour tout a } \Delta^{-}-\{0\}, \mathbb{R}_{\alpha} \subset[3, \Omega]^{\mathbb{C}} \subset d^{\mathbb{C}},
$$

Par suite,

$$
\text { R. }=S+\Lambda^{2} \quad \text { (somme non nécessajrement directe). }
$$

Désignons par $P$ le groupe de Lie connexe de $R$ ayant $P$ pour algèbre de Lie ; nous avons $R=N P$ (décomposition non nécessairement unique).

(6.2) Lemme

Pour tous éléments $r$ de $R$ et $A$ de $D(R)$, posons

$$
n(A)(r)=(\exp A u) p,
$$

où $r$ s'écrit $(\exp u) p$ avec $u \in \mathbb{L}^{\mathrm{P}}$ et $\mathrm{p} \in \mathrm{P}$.

On définit ainsi, sans ambiguité, un élément $n(A)(r)$ de $R$; $n$ est un homomorphisme continu de $D(R)$ dans le groupe des automorphismes de $\mathrm{R}$.

\section{Preuve :}

Pour tout $r \in R$, désignons par $D(r)$ la partie semi-simple de Adr; nous avons

$$
D(r) u=\sum_{\alpha \in \Delta} \phi_{\alpha}(r) u_{\alpha}, \quad(u \in R)
$$

$D(r)$ possède les propriétés suivantes :

$$
\begin{aligned}
& \text { i) } \mathrm{D}(\mathrm{r})=\mathrm{D}(\mathrm{p}) \text {, pour tout p } \mathrm{p} \mathrm{P} \text { tel que } \mathrm{rp}^{-1} \in \mathrm{N}
\end{aligned}
$$

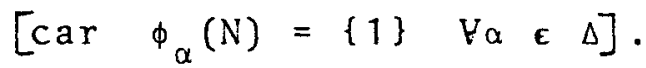


ii) $D(r) u=u, \quad \forall u \in \mathcal{N} r \Theta\left[\operatorname{car} \phi_{0} \equiv 17\right.$

iii) $D(r)$ est un automorphisme d'algèrre de Lie de 2 $\left[\operatorname{car} \phi_{\alpha+\beta}=\phi_{\alpha} \phi_{\beta}\right.$ et $\left[R_{\alpha}, R_{\beta}\right] \subset \Re_{\alpha+\beta}$, pour $\alpha, \beta \in \Delta$ avec $\alpha+\beta \in \Delta]$

iv) $D(r)$ commute avec tous les éléments de AdP [car les sous-espaces $\mathcal{B}_{\alpha}, \alpha \epsilon \Delta$, sont stables pour les éléments de $\operatorname{AdP}]$.

A l'aide de ces quatre propriétés, on vérifie aisément : tout d'abord que :

$$
(\exp A u) p=\left(\exp A u^{\prime}\right) p^{\prime}
$$

pour $A \in D(R)$ et pour $u, u^{\prime} \in \mathcal{P}, p, p^{\prime} \in \mathrm{p}$ avec $(\exp u) p=\left(\exp u^{\prime}\right) p^{\prime}$; et ensuite que $n$ est un homomorphisme continu de $D(R)$ dans le groupe des automorphismes de $R$.

Le lemme est prouvé.

Posons alors

$r_{1} \cdot r_{2}=r_{1}\left[n\left(D\left(r_{1}^{-1}\right)\right)\left(r_{2}\right)\right]$, pour tout $r_{1}, r_{2} \in R$.

On voit facilement que l'on définit ainsi un produit nilpotent sur $R$; $n$ est un homorphisme continu de $D(R)$ dans le groupe des automorphismes de $(R, \cdot)$; et $1^{\prime}$ application $R \rightarrow(R, \cdot) x_{n} D(R)$ est un isomorphisme de groupes analytiques $r \rightarrow(r, D(r))$ de $R$ sur son image.

Désignons par D(S) l'ensemble des parties semi-simples des 
éléments de ad $\Omega$; nous avons

$$
D(u) v=\sum_{\alpha \in \Delta} \alpha(u) v_{\alpha},(u, v \in \beta)
$$

Posons alors

$$
[u, v]^{\prime}=[u, v]-(D(u) v-D(v) u),(u, v \in \Re) ;
$$

puis

$[(u, A),(v, B)]^{\prime}=\left([u, v]^{\prime}+A v-B u, 0\right),(u, v \in R ; A, B \in D(\Omega))$.

$[$,$] ' est un crochet de Lie sur \Omega \oplus D\left(\mathcal{F}_{L}\right)$ et le groupe de Lie $(R, \cdot) x_{n} D(R)$ possède, $\left(\Omega \oplus D(\Omega),[,]^{\prime}\right)$ pour algèbre de Lie.

$$
\text { Le théorème }(6.1) \text { est ainsi démontré. }
$$

(6.2) Soit $G=R x_{\xi} K$ un produit semi-direct d'un groupe de Lie résoluble simplement connexe de type rigide $R$ par un groupe compact K.

Munissons l'espace produit $R \times D(R) \times K$ du produit défini par (voir lemme (6.2))

$$
\begin{aligned}
(r, D(s), k) \cdot\left(r^{\prime}, D\left(s^{\prime}\right), k^{\prime}\right)=\left(r\left[n\left(D\left(r^{-1} s\right)\right)\left(\xi(k)\left(r^{\prime}\right)\right)\right],\right. & \left.D(s) D\left(\xi(k)\left(s^{\prime}\right)\right), k k^{\prime}\right)
\end{aligned}
$$

pour $r, r^{\prime}, s, s^{\prime} \in R$ et $k, k^{\prime} \epsilon K$; nous notons $H$ le groupe ainsi obtenu. $H$ est un produit semi-direct du groupe nilpotent simplement connexe $(\mathrm{R}, \cdot)$ par un groupe compact. L'application $\psi$ de $G$ dans $H$ qui au couple $(r, k)$ associe le triplet $(r, D(r), k)$ est un isomorphisme de G sur son image.

Nous sommes ainsi ramené à la situation de la section 5 ; 
les résultats de cette section nous donnent donc un théorème de la limite centrale pour $G$.

\section{(6.3) Exemple}

Soit G le groupe constitué par l'espace produit $\mathbb{C} \times \mathbf{R}$ muni du produit

$$
(z, t)\left(z^{\prime}, t^{\prime}\right)=\left(z+e^{i t} z^{\prime}, t+t^{\prime}\right)
$$

G est le revêtement simplement connexe du groupe des déplacements du plan.

Le plongement qui résulte du théorème (6.1) est le suivant :

$$
\begin{aligned}
& \dot{\psi}: G \rightarrow H \\
& (z, t) \rightarrow\left((z, t), e^{i t}\right)
\end{aligned}
$$

où $H$ est le groupe constitué par l'espace produit $\mathbf{C} \times \mathbf{R} \times \mathrm{T}$ muni du produit

$$
\begin{aligned}
& \left(z, t, e^{i s}\right)\left(z^{\prime}, t^{\prime}, e^{i s^{\prime}}\right)=\left(z+e^{i s} z^{\prime}, t+t^{\prime}, e^{i\left(s+s^{\prime}\right)}\right) ; \\
& \text { If est donc un produit semi-direct de }\left(\mathbb{R}^{3},+\right) \text { par le tore } T .
\end{aligned}
$$

Désignons par $\lambda$ une mesure de probabilité possèdant un moment d'ordre 2 sur $G$; et par $\left\{\left(z_{i}, t_{i}\right)\right\}_{i \geq 1}$ une suite de v.a. à valeurs dans $\mathbb{C} \times \mathbf{R}$, indépendantes et de loi commune $\lambda$. Si $g$ est un élément de $G$, nous notons $z(g)=x_{1}(g)+i x_{2}(g)$ et $t(g)$ ses composantes respectivement sur $\mathbb{C}$ et $\mathbb{R}$.

$$
\text { D'après la section } 5 \text {, nous avons : }
$$

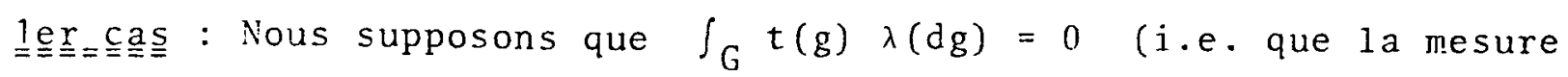
$\overline{\psi(\lambda)}$ est centrée dans $\mathrm{R}^{3}$ (notations de la section 5))'. 
Supposons que $\lambda$ ne soit pas portée par le sous-groupe C $x\{0\}$ de $G$. Désignons par a l'élément de $G$ défini par

$$
z(a)=\frac{\int_{G} z(g) \lambda(d g)}{1-\int_{G} e^{i t(g)} \lambda(d g)} \text { et } t(a)=0 \text {. }
$$

La mesure de probabilité $\varepsilon_{a^{-1}} \lambda^{*} \varepsilon_{a}$ est contrée dans $\mathbf{R}^{3}$.

Alors, d'après le théorème (5.9), la suite de v.a.

$s_{n}=\left(\frac{z_{1}+e^{i t_{1}} z_{2}+\ldots+e^{i\left(t_{1}+\ldots+t_{n-1}\right)} z_{n}}{\sqrt{n}}, \frac{t_{1}+\ldots+t_{n}}{\sqrt{n}}\right)$

converge en loj vers la loi gaussienne centrée de matrice de covariancovariance

$$
\left[\begin{array}{rrr}
\sigma_{1}^{2} & 0 & 0 \\
0 & \sigma_{1}^{2} & 0 \\
0 & 0 & \sigma_{2}^{2}
\end{array}\right] \quad, \quad \text { où }
$$

$$
\begin{aligned}
\sigma_{1}^{2} & =\int_{G}|z(g)|^{2} \varepsilon_{a}{ }^{-1} \lambda * \varepsilon_{a}(d g) \\
& =\int_{G}\left|z(g)-\frac{1-e^{i t(g)}}{1-\int_{G} e^{i t(g)} \lambda(d g)} \int_{G} z(g) \lambda(d g)\right|^{2} \lambda(d g)
\end{aligned}
$$

et

$\sigma_{2}^{2}=\operatorname{var} t_{1}=\int_{G} t^{2}(g) \lambda(d g)$

Cette loi limite est non dégénérée si et seulement si $\lambda$ n'est portêe ni par $C \times\{0\}$, ni par a $(\{0\} \times R) a^{-1}$.

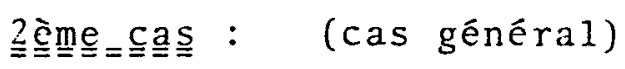

Désignons par $\tau$ l'élément de $G$ défini par' $z(\tau)=0$ et $t(\tau)=\int_{G} t(g) \lambda(d g)$. 
Supposons que la mesure $\lambda * \varepsilon_{\tau}-1$ ne soit pas portée par le sousgroupe $\mathbb{C} \times\{0\}$ de $G$ et notons a l'élément de $G$ défini par $z(a)=\frac{\int_{G} z(g) \lambda * \varepsilon_{T}-1(d g)}{1-\int_{G} e^{i t(g)} \lambda_{t * \varepsilon_{T}-1}(d g)} \quad, \quad t(a)=0$.

La mesure de probabilité $\varepsilon_{a^{-1}} * \lambda * \varepsilon_{\tau}-1$ a est centrée dans $R^{3}$. Alors, d'après le théorème (5.15), la suite de v.a.

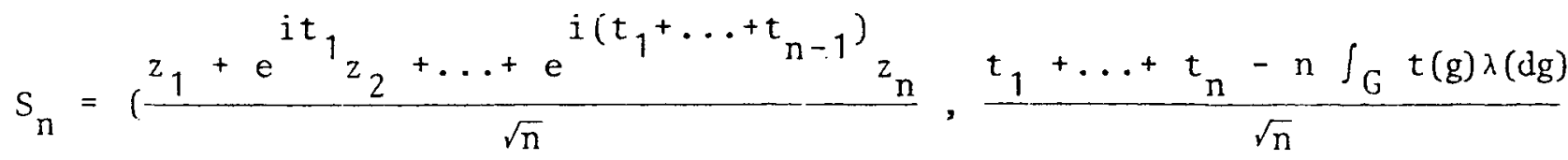
converge en $10 \mathrm{i}$ vers la loi gaussienne centrée de matrice de covariance $\quad\left[\begin{array}{ccc}\sigma_{1}^{2} & 0 & 0 \\ 1 & & \\ 0 & \sigma_{1}^{2} & 0 \\ 0 & 0 & \sigma_{2}^{2}\end{array}\right] \quad, \quad$ où

$\sigma_{1}^{2}=\int_{G}|z(g)|^{2} \varepsilon_{a}-1 * \lambda * \varepsilon_{\tau}-1 \frac{d g}{a}(d g)$

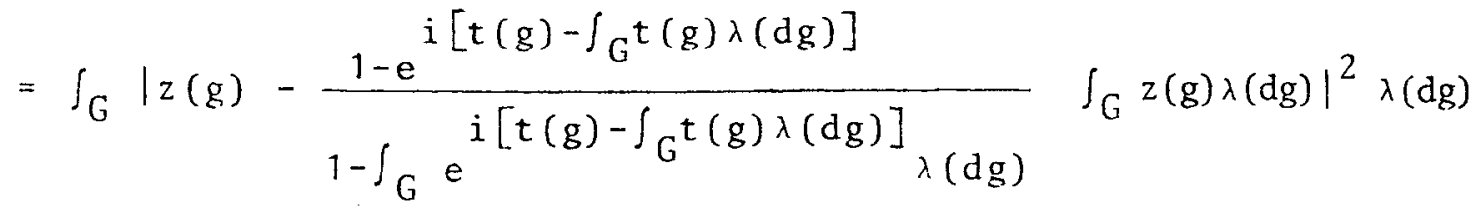

et $\sigma_{2}^{2}=\operatorname{var} t_{1}=\int_{G} t^{2}(g) \lambda(d g)-\left(\int_{G} t(g) \lambda(d g)\right)^{2}$.

Cette loi limite est non dégénérée si et seulement si $\lambda$ n'est portée ni par $\mathbb{C} \times\{\tau\}$, ni par a $(\{0\} \times \mathbb{R}) \mathrm{a}^{-1} \tau$. 
[1] DERRIENNIC Y. : "Lois "zéro ou deux" pour les processus de Markov. Applications aux marches aléatoires". Ann. Inst. Henri Poincarré, vol XII, $\mathrm{n}^{\circ} 2,1976, \mathrm{p} .111-129$.

[2] GUIVARC'H Y. : "Loi des grands nombres et rayon spectral pour une marche aléatoire sur un groupe de Lie". Bull. Soc. math. France, (à paraître).

[3] GUIVARC'H Y. : "Croissance polynomiale et périodes des fonctions harmoniques". Bull. Soc. Math. France, 101, 1973, 333-379.

[4] ICHIHARA K., KUNITA $H$. : "A classification of the second order degenerate elliptic operators and its probabilistic characterization". Zeit. für Wahr. 30, 1974, p.235.

[5] RAUGI A. : "Théorème de la limite centrale pour les groupes de Lie nilpotents simplement connexes". Zeit. für Wahr. (à parầtre).

[6] RAUGI A. : "Fonctions harmoniques et théorèmes limites pour les marches aléatoires sur les groupes". Bull. Soc. Math. France, Mémoire $54,1977,127 \mathrm{p}$.

[7] SAZONOV V.V. and TUTUBALIN V.N. "Probability distributions on topological groups". Theory Prob. Applications, 11(1966), pp. 3-55.

[8] SKOROKHOD A.V. : "Studies in the theory of Random Process", Addison-liesley publishing company, Inc. Massachussets, 1965. 